\title{
Unique continuation inequalities for the parabolic-elliptic chemotaxis system*
}

\author{
Gengsheng Wang ${ }^{a}$, Guojie Zheng ${ }^{b}$ \\ ${ }^{a}$ Center for Applied Mathematics \\ Tianjin University, Tianjin 300072, P.R. China \\ ${ }^{b}$ College of Mathematics and Information Science \\ Henan Normal University, Xinxiang, 453007, P.R. China
}

\begin{abstract}
This paper studies the quantitative unique continuation for a semi-linear parabolic-elliptic coupled system on a bounded domain $\Omega$. This system is a simplified version of the chemotaxis model introduced by Keller and Segel in 14. With the aid of priori $L^{\infty}$-estimates (for solutions of the system) built up in this paper, we treat the semi-linear parabolic equation in the system as a linear parabolic equation, and then use the frequency function method and the localization technique to build up two unique continuation inequalities for the system. As a consequence of the above-mentioned two inequalities, we have the following qualitative unique continuation property: if one component of a solution vanishes in a nonempty open subset $\omega \subset \Omega$ at some time $T>0$, then the solution is identically zero.
\end{abstract}

Keywords: Unique continuation inequalities; parabolic-elliptic coupled system; frequency function method; localization technique

AMS subject classifications: $35 \mathrm{~K} 20,35 \mathrm{~K} 55$.

\section{Introduction}

This paper studies the unique continuation property for the semi-linear parabolic-elliptic coupled system:

$$
\begin{cases}\partial_{t} u(x, t)-\Delta u(x, t)+\nabla \cdot(u(x, t) \nabla v(x, t))=0, & \text { in } \Omega \times(0,+\infty), \\ -\triangle v(x, t)+a v(x, t)-b u(x, t)=0, & \text { in } \Omega \times(0,+\infty), \\ u(x, t)=0, v(x, t)=0, & \text { on } \partial \Omega \times(0,+\infty), \\ u(x, 0)=u_{0}(x), & \text { in } \Omega,\end{cases}
$$

${ }^{*}$ The authors were supported by the NNSF of China under grants 11971022, 11801408, and by the Natural Science Foundation of Henan Province 202300410248. E-mail addresses: wanggs62@yeah.net (Gengsheng Wang); guojiezheng@yeah.net (Guojie Zheng). 
where $\Omega$ is a bounded domain in $\mathbb{R}^{n}(n \geq 1)$ with a $C^{2}$ boundary $\partial \Omega, a$ and $b$ are positive constants, $u_{0}$ is an initial datum. The system (1.1) is a simplified version of the chemotaxis model introduced by Keller and Segel in [14, which depicts the change of motion when a population reacts in response to an external chemical stimulus spread in the environment where they reside. In the system (1.1), $u$ and $v$ stand for the concentration of species and the chemical substance, respectively. (See [12].) A slightly generalized model of (1.1) consists of two parabolic equations:

$$
\begin{cases}\partial_{t} u(x, t)=\Delta u(x, t)-\nabla \cdot(u(x, t) \nabla v(x, t)), & \text { in } \Omega \times(0,+\infty), \\ \tau \partial_{t} v(x, t)=\Delta v(x, t)-a v(x, t)+b u(x, t), & \text { in } \Omega \times(0,+\infty),\end{cases}
$$

with the time scale $\tau<<1$, which means that the time scale of the chemical diffusion is shorter than that of species. (See [11].) We refer readers to [12, 13] for more background about chemotaxis and its model. In the studies of the chemotaxis model, boundary conditions can be either Dirichlet type, Neumann type, or Robin type, particularly, the homogeneous Dirichlet boundary condition means the zero density on the boundary. (See [15].) This kind of Keller-Segel system was studied in [6, 9, 16, where the local existence, the global existence, and the blow-up phenomenon of solutions were obtained.

This paper aims to show two unique continuation inequalities for the system (1.1), from which, one can directly get the following qualitative unique continuation property: if one component of a solution vanishes in a nonempty open subset $\omega \subset \Omega$ at some time $T>0$, then the solution is identically zero.

Most studies on the unique continuation property for PDEs focus on the linear cases. In [21], the authors reduced parabolic equations (with constant coefficients) to elliptic forms. The technique developed in [21] also works for parabolic equations with coefficients depending only on the spacevariable. The unique continuation property of parabolic equations with potential was built up in [22, in which the order of the solution's vanishing at some interior point was investigated. We also mention [28] where some weak unique continuation property was obtained. In studies of the quantitative unique continuation property for linear parabolic equations, the Carleman inequality and the frequency function are two important tools (see, for instance, [8]). About the Carleman estimates, we would like to mention works [4, 7, 17, 19]. The frequency function for elliptic equations may be traced back to [2]. A slightly different version of this type of function for parabolic equations was used in [24] and 25], where some unique continuation inequalities were obtained. We also mention [10, 20, 23, 26, 27] for the related studies. To the best of our knowledge, there are very limited works on the unique continuation property for nonlinear equations. We mention [18] and [30] in this direction. In [30], the author used the inverse scattering theory to obtain the unique continuation property for the Korteweg-de Vries equation.

The main theorem of this paper is as follows:

Theorem 1.1. Let $u_{0} \in L^{\infty}(\Omega)$ and let $\omega$ be a nonempty open subset of $\Omega$. Suppose that $(u, v)$ is the solution to the system (1.1) over $[0, T]$ for some $T>0$. Then the following conclusions are true:

(i) There are $\gamma=\gamma\left(\Omega, \omega,\left\|u_{0}\right\|_{L^{\infty}(\Omega)}, T\right) \in(0,1)$ and $D=D\left(\Omega, \omega,\left\|u_{0}\right\|_{L^{\infty}(\Omega)}, T\right)>0$ so that

$$
\int_{\Omega}\left(|u(x, T)|^{2}+|v(x, T)|^{2}\right) d x \leq D\left(\int_{\Omega}\left|u_{0}(x)\right|^{2} d x\right)^{1-\gamma}\left(\int_{\omega}|u(x, T)|^{2} d x\right)^{\gamma} .
$$


(ii) When $u_{0} \neq 0$, there is $C=C\left(\Omega, \omega,\left\|u_{0}\right\|_{L^{\infty}(\Omega)}, T\right)>0$ so that

$$
\int_{\Omega}\left|u_{0}(x)\right|^{2} d x \leq C \exp \left(C \frac{\left\|u_{0}(x)\right\|_{L^{2}(\Omega)}^{2}}{\left\|u_{0}(x)\right\|_{H^{-1}(\Omega)}^{2}}\right) \times \int_{\omega}\left(|u(x, T)|^{2}\right) d x .
$$

Remark 1.1. Several notes on Theorem 1.1 are given in order.

(a1) Our motivations to build up Theorem 1.1 are as follows: From the perspective of mathematics, most studies on the unique continuation property focus on linear systems, while (1.1) is a semi-linear system. From the application point of view, one can recover the initial population state and evolution history from the observation in a small subset at a time, through using our unique continuation inequalities.

(a2) From either (1.2) or (1.3), we can get the following qualitative unique continuation property for the system (1.1): if $u(x, T)=0$ over $\omega$ or $v(x, T)=0$ over $\omega$, then $(u, v)$ is identically zero. (See Corollary 4.1.) Moreover, (1.3) implies the backward uniqueness: if $u(\cdot, T)=0$ over $\Omega$, then $u_{0}=0$.

(a3) It is natural to ask if (1.2) or (1.3) still holds when $\int_{\omega}\left(|u(x, T)|^{2}\right) d x$ is replaced by $\int_{\omega}\left(|v(x, T)|^{2}\right) d x$ on the right hand side. Unfortunately, we are not able to answer this question now.

(a4) Our strategy to show Theorem 1.1 is as follows: We rewrite (1.1) as:

$$
\partial_{t} u-\triangle u+A \cdot \nabla u+B u=0
$$

where $A=A(v)$ and $B=B(u, v)$ depend on $u$ and $v$. (We can do this because of the structure of (1.1).) The equation (1.4) hints us to build up priori $L^{\infty}$-estimates for the solution $(u, v)$ to the system (1.1) with $u_{0} \in L^{\infty}(\Omega)$, which gives $L^{\infty}$-estimates of $A$ and $B$ in terms of $\left\|u_{0}\right\|_{L^{\infty}(\Omega)}$. Then we treat (1.4) as a linear parabolic equation where $A$ and $B$ are viewed as coefficients with the above-mentioned $L^{\infty}$-estimates. After that, we use the frequency function method (see, for instance, [3, 23, 24, 26]) to prove a local interpolation inequality (see Theorem [3.1). Finally, we utilize the localization technique (see [25]) to get Theorem [1.1.

It seems to us that the above strategy might be used to build up the unique continuation inequalities for some more general semi-linear parabolic equations.

The rest of the paper is organized as follows: Section 2 presents the well-posedness and some estimates for the system (1.1). Section 3 shows a local interpolation inequality of the system (1.1). Section 4 proves Theorem 1.1 .

\section{Analysis of the system (1.1)}

We start with introducing notation. We use $\|\cdot\|_{p}($ with $(p \in[1,+\infty]))$ to denote the norm of the space $L^{p}(\Omega)$. We use $B\left(x_{0}, R\right)$ to stand for the open ball centered at $x_{0}$ and of radius $R$, and use $C(\ldots)$ to stand for a positive constant which depends on what are enclosed in the brackets. 


\subsection{Preliminary lemmas}

We introduce several estimates on a linear elliptic equation:

$$
\begin{cases}-\triangle v(x)+\hat{a} v(x)=\eta(x), & \text { in } \Omega, \\ v(x)=0, & \text { on } \partial \Omega,\end{cases}
$$

(Here, $\hat{a}$ is a positive constant and $\eta$ is a given function.) and on the semigroup $S_{p}(t)$ generated by $A_{p}$ on $L^{p}(\Omega)$, with $p \in(1,+\infty)$, where

$$
A_{p}(u):=\triangle u, \quad u \in W^{2, p}(\Omega) \cap W_{0}^{1, p}(\Omega) .
$$

The following two lemmas can be found in [1] and will be used later.

Lemma 2.1. For each $p \in(1,+\infty)$ and each $\eta \in L^{p}(\Omega)$, the equation (2.1) has a unique solution $v \in W^{2, p}(\Omega) \cap W_{0}^{1, p}(\Omega)$. Moreover, there is $C=C(\Omega, p, \hat{a})$ so that

$$
\|v\|_{W^{2, p}(\Omega)} \leq C\|\eta\|_{p}
$$

Lemma 2.2. For each $p \in(1,+\infty)$, there is $C=C(\Omega, p)$ so that for each $\varphi \in L^{p}(\Omega)$,

$$
\left\|S_{p}(t) \varphi\right\|_{p} \leq\|\varphi\|_{p} \text { and }\left\|\nabla S_{p}(t) \varphi\right\|_{p} \leq C t^{-\frac{1}{2}}\|\varphi\|_{p}, \text { when } t \in(0,+\infty) .
$$

As a direct consequence of Lemma 2.1, we have

Corollary 2.1. For each $p \in(1,+\infty)$, the resolvent $J_{\hat{a}}:=(-\triangle+\hat{a} I)^{-1}$ (with $I$ the identity operator on $\left.L^{p}(\Omega)\right)$ is a linear bounded operator from $L^{p}(\Omega)$ to $W^{2, p}(\Omega) \cap W_{0}^{1, p}(\Omega)$. Moreover, for each $\eta \in L^{p}(\Omega)$, the solution of (2.1) satisfies $v=J_{\hat{a}}(\eta)$.

Though the divergence operator does not commute with $S_{p}(t)(t>0)$, the next Lemma 2.3 remains true.

Lemma 2.3. For each $p \in(1,+\infty)$, there is $C=C(\Omega, p)$ so that for each $\Phi \in\left[C_{0}^{\infty}(\Omega)\right]^{n}$,

$$
\left\|S_{p}(t) \nabla \cdot \Phi\right\|_{p} \leq C(\Omega) t^{-\frac{1}{2}}\|\Phi\|_{\left[L^{p}(\Omega)\right]^{n}}, \text { when } t \in(0,+\infty) .
$$

Proof. Let $p^{\prime} \in(1,+\infty)$ satisfy $\frac{1}{p}+\frac{1}{p^{\prime}}=1$. Then, it follows from Lemma 2.2 that when $t \in(0,+\infty)$,

$$
\begin{aligned}
& \left\|S_{p}(t) \nabla \cdot \Phi\right\|_{p}=\sup _{\|\phi\|_{p^{\prime}}=1}\left|\left\langle S_{p}(t) \nabla \cdot \Phi, \phi\right\rangle_{p, p^{\prime}}\right| \\
= & \sup _{\|\phi\|_{p^{\prime}}=1}\left|\left\langle\Phi, \nabla S_{p^{\prime}}(t) \phi\right\rangle_{\left[L^{p}(\Omega)\right]^{n},\left[L^{p^{\prime}}(\Omega)\right]^{n}}\right| \leq C(\Omega) t^{-\frac{1}{2}}\|\Phi\|_{\left[L^{p}(\Omega)\right]^{n} .}
\end{aligned}
$$

(Here, $\langle\cdot, \cdot\rangle_{p, p^{\prime}}$ denotes the pair between $L^{p}(\Omega)$ and $L^{p^{\prime}}(\Omega)$ and $\langle\cdot, \cdot\rangle_{\left[L^{p}(\Omega)\right]^{n},\left[L^{p^{\prime}}(\Omega)\right]^{n}}$ stands for the pair between $\left[L^{p}(\Omega)\right]^{n}$ and $\left[L^{p^{\prime}}(\Omega)\right]^{n}$.) This completes the proof.

Remark 2.1. By Lemma 2.3, we can use the standard density argument to see that for each $p \in(1,+\infty)$ and each $t>0$, the operator $S_{p}(t) \nabla \cdot$ has a unique extension over $\left[L^{p}(\Omega)\right]^{n}$, which will be denoted in the same manner. Thus, (2.2) holds for all $\Phi \in\left[L^{p}(\Omega)\right]^{n}$. 
We end this subsection by introducing an estimate on the initial-boundary value problem:

$$
\begin{cases}\partial_{t} y(x, t)=\mathcal{A}(t) y(x, t), & \text { in } \Omega \times(0, \bar{T}], \\ y(x, t)=0, & \text { on } \partial \Omega \times(0, \bar{T}], \\ y(x, 0)=y_{0}(x), & \text { in } \Omega .\end{cases}
$$

Here, $\bar{T}>0$ is arbitrarily fixed and $\mathcal{A}(t)$ is the differential operator defined by

$$
\mathcal{A}(t) y:=\sum_{i, j=1}^{n} \frac{\partial}{\partial x_{i}}\left(a_{i j}(x, t) \frac{\partial y}{\partial x_{j}}\right)+\sum_{i=1}^{n} b_{i}(x, t) \frac{\partial y}{\partial x_{i}}+c(x, t) y,
$$

with the real-valued coefficient functions $a_{i j}, b_{i}, c \in L^{\infty}(\Omega \times(0, \bar{T}]),(i, j=1, \ldots, n)$. Moreover, we assume that $\mathcal{A}(t)$ is uniformly strongly elliptic, that is, there exists $\alpha_{0}>0$ such that for a.e. $(x, t) \in \Omega \times(0, \bar{T}]$

$$
\sum_{i, j=1}^{n} a_{i j}(x, t) \xi_{i} \xi_{j} \geq \alpha_{0}|\xi|^{2} \text { for all } \xi=\left(\xi_{1}, \xi_{2}, \ldots, \xi_{n}\right) \in \mathbb{R}^{n}
$$

Lemma 2.4. Let $U(t, s)(\bar{T} \geq t \geq s \geq 0)$ be the evolution system generated by $\mathcal{A}(t)(\bar{T} \geq t \geq 0)$. Then there is a positive constant $\varpi:=\varpi\left(\Omega, n, L, \alpha_{0}\right)$, where

$$
L=\max \left\{1,\left\|b_{i}\right\|_{L^{\infty}(\Omega \times(0, \bar{T}))},\|c\|_{L^{\infty}(\Omega \times(0, \bar{T}))} \mid i=1, \ldots, n\right\},
$$

and $\alpha_{0}$ is given by (2.3), so that when $1 \leq p \leq q \leq+\infty$ and $y_{0} \in L^{p}(\Omega)$,

$$
\left\|U(t, s) y_{0}\right\|_{q} \leq e^{\varpi[1+(t-s)]}(t-s)^{-\frac{n}{2}\left(\frac{1}{p}-\frac{1}{q}\right)}\left\|y_{0}\right\|_{p}, \text { for } \bar{T} \geq t>s \geq 0 .
$$

Remark 2.2. We quote Lemma 2.4 from [5], which doesn't give what quantities the constants in the inequality (2.4) depend on. However, following the proof in [5], we can get them.

\subsection{An auxiliary system}

In this subsection, we study the following auxiliary system of (1.1):

$$
\begin{cases}\partial_{t} u(x, t)-\triangle u(x, t)+\nabla \cdot(u(x, t) \nabla v(x, t))=0, & \text { in } \Omega \times(0,+\infty), \\ -\triangle v(x, t)+a v(x, t)-b \xi(x, t)=0, & \text { in } \Omega \times(0,+\infty), \\ u(x, t)=0, v(x, t)=0, & \text { on } \partial \Omega \times(0,+\infty), \\ u(x, 0)=u_{0}(x), & \text { in } \Omega,\end{cases}
$$

where $\xi \in L^{\infty}\left(0,+\infty ; L^{p}(\Omega)\right)$ with $n<p<+\infty$.

Proposition 2.1. Given $u_{0} \in L^{p}(\Omega), \xi \in L^{\infty}\left(0,+\infty ; L^{p}(\Omega)\right.$ ) (with $p \in(n,+\infty)$ ) and $\bar{T}>0$, the system (2.5) has a unique solution $(u, v)$ over $[0, \bar{T}]$. Moreover, $(u, v)$ belongs to $C\left([0, \bar{T}] ; L^{p}(\Omega)\right) \times$ $L^{\infty}\left(0, \bar{T} ; W^{2, p}(\Omega) \cap W_{0}^{1, p}(\Omega)\right)$ and satisfies that for some $C:=C\left(\Omega, \bar{T},\|\xi\|_{L^{\infty}\left(0, \bar{T} ; L^{p}(\Omega)\right)}\right)>0$,

$$
\|u\|_{C\left([0, \bar{T}] ; L^{p}(\Omega)\right)} \leq C\left\|u_{0}\right\|_{p}
$$

If we further assume that $\xi \in C\left([0,+\infty) ; L^{p}(\Omega)\right)$, then $(u, v) \in C\left([0, \bar{T}] ; L^{p}(\Omega)\right) \times C\left((0, \bar{T}] ; W^{2, p}(\Omega) \cap\right.$ $\left.W_{0}^{1, p}(\Omega)\right)$. 
Proof. Arbitrarily fix $p \in(n,+\infty), u_{0} \in L^{p}(\Omega), \xi \in L^{\infty}\left(0,+\infty ; L^{p}(\Omega)\right)$ and $\bar{T}>0$. Two observations are given in order. First, according to Corollary 2.1,

$$
v(t)=J_{a}(b \xi(t)), \quad t \in(0, \bar{T}],
$$

and $J_{a} \in \mathcal{L}\left(L^{p}(\Omega) ; W^{2, p}(\Omega) \cap W_{0}^{1, p}(\Omega)\right)$, where $v$ is the unique solution to the second equation of (2.5). From these and the fact that $\xi \in L^{\infty}\left(0,+\infty ; L^{p}(\Omega)\right)$, it follows from the standard method in [29] that $v \in L^{\infty}\left(0, \bar{T} ; W^{2, p}(\Omega) \cap W_{0}^{1, p}(\Omega)\right)$. (See Section V of [29], p.134.) Then by the Sobolev embedding theorem and Lemma 2.1, we have

$$
\begin{aligned}
& \|v\|_{L^{\infty}\left(0, \bar{T} ; L^{\infty}(\Omega)\right)}+\|\nabla v\|_{L^{\infty}\left(0, \bar{T} ;\left[L^{\infty}(\Omega)\right]^{n}\right)} \\
\leq & C(\Omega)\|v\|_{L^{\infty}\left(0, \bar{T} ; W^{2, p}(\Omega) \cap W_{0}^{1, p}(\Omega)\right)} \leq C(\Omega)\|\xi\|_{L^{\infty}\left(0, \bar{T} ; L^{p}(\Omega)\right)} .
\end{aligned}
$$

Second, we arbitrarily fix $\eta \in C\left([0, \bar{T}] ; L^{p}(\Omega)\right)$. Then it follows from Lemma 2.2 and Lemma 2.3 that for each $t \in[0, \bar{T}]$,

$$
\begin{aligned}
& \left\|S_{p}(t) u_{0}-\int_{0}^{t} S_{p}(t-s) \nabla \cdot(\eta \nabla v) d s\right\|_{p} \\
\leq & \left\|u_{0}\right\|_{p}+C(\Omega) \int_{0}^{t}(t-s)^{-\frac{1}{2}}\|\eta \nabla v\|_{\left[L^{p}(\Omega)\right]^{n}} d s \\
\leq & \left\|u_{0}\right\|_{p}+C(\Omega) \bar{T}^{\frac{1}{2}}\|\eta\|_{C\left([0, \bar{T}] ; L^{p}(\Omega)\right)}\|\nabla v\|_{L^{\infty}\left(0, \bar{T} ;\left[L^{\infty}(\Omega)\right]^{n}\right)} .
\end{aligned}
$$

Now by (2.9) and (2.8), we see that for each $t \in[0, \bar{T}]$,

$$
\left\|S_{p}(t) u_{0}-\int_{0}^{t} S_{p}(t-s) \nabla \cdot(\eta \nabla v) d s\right\|_{p} \leq\left\|u_{0}\right\|_{p}+C(\Omega) \bar{T}^{\frac{1}{2}}\|\eta\|_{C\left([0, \bar{T}] ; L^{p}(\Omega)\right)}\|\xi\|_{L^{\infty}\left(0, \bar{T} ; L^{p}(\Omega)\right)} .
$$

This yields $F(\cdot ; \eta) \in C\left([0, \bar{T}] ; L^{p}(\Omega)\right)$, where

$$
F(t ; \eta):=S_{p}(t) u_{0}-\int_{0}^{t} S_{p}(t-s) \nabla \cdot(\eta \nabla v) d s, \quad t \in[0, \bar{T}] .
$$

Thus, we can define a map $\Lambda: C\left([0, \bar{T}] ; L^{p}(\Omega)\right) \mapsto C\left([0, \bar{T}] ; L^{p}(\Omega)\right)$ in the following manner: for each $\eta \in C\left([0, \bar{T}] ; L^{p}(\Omega)\right)$, set

$$
\Lambda(\eta)(t):=F(t ; \eta), t \in[0, \bar{T}]
$$

We are going to finish the proof with the aid of the contraction mapping theorem. To this end, we see from Lemma 2.3 that when $\eta_{1}, \eta_{2} \in C\left([0, \bar{T}] ; L^{p}(\Omega)\right)$,

$$
\begin{aligned}
\left\|\Lambda\left(\eta_{1}\right)-\Lambda\left(\eta_{2}\right)\right\|_{C\left([0, \bar{T}] ; L^{p}(\Omega)\right)} & \leq \int_{0}^{t}\left\|S_{p}(t-s) \nabla \cdot\left(\left(\eta_{1}-\eta_{2}\right) \nabla v\right)\right\| d s \\
& \leq \int_{0}^{t} C(\Omega)(t-s)^{-\frac{1}{2}}\left\|\left(\eta_{1}-\eta_{2}\right) \nabla v\right\|_{\left[L^{p}(\Omega)\right]^{n}} d s .
\end{aligned}
$$

This, along with (2.8), implies

$$
\left\|\Lambda\left(\eta_{1}\right)-\Lambda\left(\eta_{2}\right)\right\|_{C\left([0, \bar{T}] ; L^{p}(\Omega)\right)} \leq C(\Omega) \bar{T}^{\frac{1}{2}}\|\xi\|_{L^{\infty}\left(0, \bar{T} ; L^{p}(\Omega)\right)}\left\|\eta_{1}-\eta_{2}\right\|_{C\left([0, \bar{T}] ; L^{p}(\Omega)\right)} .
$$


We first consider the case that

$$
C(\Omega) \bar{T}^{\frac{1}{2}}\|\xi\|_{L^{\infty}\left(0, \bar{T} ; L^{p}(\Omega)\right)}<1 .
$$

From (2.10) and (2.11), we see that $\Lambda$ is a strict contraction map. Thus it has a unique fixed point $u \in C\left([0, \bar{T}] ; L^{p}(\Omega)\right)$, i.e.,

$$
u(t)=S_{p}(t) u_{0}-\int_{0}^{t} S_{p}(t-s) \nabla \cdot(u \nabla v) d s, \quad t \in[0, \bar{T}] .
$$

Consequently, $u \in C\left([0, \bar{T}] ; L^{p}(\Omega)\right)$ is the unique solution to the first equation of (2.5) (corresponding to the above $v)$ over $[0, \bar{T}]$. Hence, $(u, v) \in C\left([0, \bar{T}] ; L^{p}(\Omega)\right) \times L^{\infty}\left(0, \bar{T} ; W^{2, p}(\Omega) \cap W_{0}^{1, p}(\Omega)\right)$ is the unique solution to (2.5) over $[0, \bar{T}]$ in the case (2.11). Meanwhile, one can easily check that $\Lambda(0)=S_{p}(t) u_{0} \in C\left([0, \bar{T}] ; L^{p}(\Omega)\right)$. By these, we can take $\eta_{1}=u$ and $\eta_{2}=0$ in (2.10) to get

$$
\begin{aligned}
& \|u\|_{C\left([0, \bar{T}] ; L^{p}(\Omega)\right)}=\|\Lambda(u)\|_{C\left([0, \bar{T}] ; L^{p}(\Omega)\right)} \\
\leq & \left\|S_{p}(t) u_{0}\right\|_{C\left([0, \bar{T}] ; L^{p}(\Omega)\right)}+\|\Lambda(u)-\Lambda(0)\|_{C\left([0, \bar{T}] ; L^{p}(\Omega)\right)} \\
\leq & \left\|u_{0}\right\|_{p}+C(\Omega) \bar{T}^{\frac{1}{2}}\|\xi\|_{L^{\infty}\left(0, \bar{T} ; L^{p}(\Omega)\right)}\|u\|_{C\left([0, \bar{T}] ; L^{p}(\Omega)\right)},
\end{aligned}
$$

which leads to (2.6) for the case (2.11).

We next consider the case that

$$
C(\Omega) \bar{T}^{\frac{1}{2}}\|\xi\|_{L^{\infty}\left(0, \bar{T} ; L^{p}(\Omega)\right)} \geq 1
$$

By using a standard iteration argument (by choosing another $\bar{T}_{1}>0$ with $C(\Omega) \bar{T}_{1}^{\frac{1}{2}}\|\xi\|_{L^{\infty}\left(0, \bar{T} ; L^{p}(\Omega)\right)}<$ 1 ) and the results obtained in the case (2.11), we can get the desired results for the case (2.12).

Finally, we assume that $\xi \in C\left([0,+\infty) ; L^{p}(\Omega)\right)$. Then it follows from (2.7), the linearity and boundedness of $J_{a}$ that

$$
\begin{aligned}
& \left\|v\left(t_{1}\right)-v\left(t_{2}\right)\right\|_{W^{2, p}(\Omega)}=\left\|b J_{a}\left(\xi\left(t_{1}\right)-\xi\left(t_{2}\right)\right)\right\|_{W^{2, p}(\Omega)} \\
\leq & b\left\|J_{a}\right\| \cdot\left\|\xi\left(t_{1}\right)-\xi\left(t_{2}\right)\right\|_{p}, \quad \text { when } t_{1}, t_{2} \in(0,+\infty) .
\end{aligned}
$$

This, along with the continuity of $\xi$, yields that $(u, v) \in C\left([0, \bar{T}] ; L^{p}(\Omega)\right) \times C\left((0, \bar{T}] ; W^{2, p}(\Omega) \cap\right.$ $\left.W_{0}^{1, p}(\Omega)\right)$.

Hence, we finish the proof.

\subsection{Well-posedness and estimates on the system (1.1)}

In this subsection, we first build up the well-posedness for the system (1.1), then we present some estimates for the solution to the system (1.1).

Theorem 2.1. Let $n<p<+\infty$ and $u_{0} \in L^{p}(\Omega)$. Then the following conclusions are true:

(i) The system (1.1) has a unique solution $(u, v) \in C\left(\left[0, T^{*}\right] ; L^{p}(\Omega)\right) \times C\left(\left(0, T^{*}\right] ; W^{2, p}(\Omega) \cap W_{0}^{1, p}(\Omega)\right)$ for some positive constant $T^{*}:=T^{*}\left(\left\|u_{0}\right\|_{p}, \Omega\right)$.

(ii) The above solution satisfies $\left(t^{\frac{n}{2 p}} u, v\right) \in\left[L^{\infty}\left(0, T^{*} ; L^{\infty}(\Omega)\right)\right]^{2}$.

(iii) If we further assume that $u_{0} \in L^{\infty}(\Omega)$, then $(u, v) \in\left[L^{\infty}\left(0, T^{*} ; L^{\infty}(\Omega)\right)\right]^{2}$. 
Proof. Arbitrarily fix $n<p<+\infty$ and $u_{0} \in L^{p}(\Omega)$. We organize the proof in several steps.

Step 1. We prove that for some $T^{*}:=T^{*}\left(\left\|u_{0}\right\|_{p}, \Omega\right)>0$, the system (1.1) has a solution $(u, v) \in$ $C\left(\left[0, T^{*}\right] ; L^{p}(\Omega)\right) \times C\left(\left(0, T^{*}\right] ; W^{2, p}(\Omega) \cap W_{0}^{1, p}(\Omega)\right)$ satisfying

$$
\|u\|_{C\left(\left[0, T^{*}\right] ; L^{p}(\Omega)\right)} \leq C\left(\left\|u_{0}\right\|_{p}, T^{*}, \Omega\right) .
$$

We will use the contraction mapping theorem to prove it. To this end, we first set up the following framework: Let $T^{*}>0$, which will be determined later. Let

$$
K:=\left\{\xi \in C\left(\left[0, T^{*}\right] ; L^{p}(\Omega)\right) \mid\|\xi\|_{C\left(\left[0, T^{*}\right] ; L^{p}(\Omega)\right)} \leq 2\left\|u_{0}\right\|_{p}\right\}
$$

According to Proposition 2.1, for each $\xi \in K$, the system (2.5) (with the above $u_{0}$ and the zero extension of $\xi$ over $\left.\left(T^{*},+\infty\right)\right)$ has a unique solution $\left(u^{\xi}, v^{\xi}\right) \in C\left(\left[0, T^{*}\right] ; L^{p}(\Omega)\right) \times C\left(\left(0, T^{*}\right] ; W^{2, p}(\Omega) \cap\right.$ $\left.W_{0}^{1, p}(\Omega)\right)$. We define a map $\Psi$ from $K$ to $C\left(\left[0, T^{*}\right] ; L^{p}(\Omega)\right)$ by setting

$$
\Psi(\xi):=u^{\xi} \text { for each } \xi \in K
$$

We claim that for some $T^{*}>0, \Psi$ has a unique fixed point $u$ in $K$. When this is done, we have

$$
u(t)=S_{p}(t) u_{0}-\int_{0}^{t} S_{p}(t-s) \nabla \cdot(u \nabla v) d s, t \in\left[0, T^{*}\right]
$$

and

$$
\begin{cases}-\triangle v+a v-b u=0, & \text { in } \Omega \times\left(0, T^{*}\right], \\ v=0, & \text { on } \partial \Omega \times\left(0, T^{*}\right] .\end{cases}
$$

From these, we see that $(u, v):=\left(u, v^{u}\right) \in C\left(\left[0, T^{*}\right] ; L^{p}(\Omega)\right) \times C\left(\left(0, T^{*}\right] ; W^{2, p}(\Omega) \cap W_{0}^{1, p}(\Omega)\right)$ is a solution to the system (1.1).

We now check conditions ensuring the contraction mapping theorem. First, it is clear that $K$ is a closed subset of $C\left(\left[0, T^{*}\right] ; L^{p}(\Omega)\right)$. Second, we claim that $\Psi(K) \subseteq K$ for some $T^{*}>0$. Indeed, it follows from Lemma 2.2 and Lemma 2.3 that

$$
\begin{aligned}
&\left\|u^{\xi}(t)\right\|_{p} \leq\left\|S_{p}(t) u_{0}\right\|_{p}+\left\|\int_{0}^{t} S_{p}(t-s) \nabla \cdot\left(u^{\xi} \nabla v^{\xi}\right) d s\right\|_{p} \\
& \leq\left\|u_{0}\right\|_{p}+C(\Omega) t^{\frac{1}{2}}\left\|\nabla v^{\xi}\right\|_{L^{\infty}\left(0, T^{*} ;\left[L^{\infty}(\Omega)\right]^{n}\right)}\left\|u^{\xi}\right\|_{C\left(\left[0, T^{*}\right] ; L^{p}(\Omega)\right)} \text { for each } t \in\left[0, T^{*}\right] .
\end{aligned}
$$

This, together with (2.8) and the fact $\xi \in K$, yields

$$
\begin{aligned}
& \left\|u^{\xi}(t)\right\|_{p} \leq\left\|u_{0}\right\|_{p}+C(\Omega) t^{\frac{1}{2}}\|\xi\|_{C\left(\left[0, T^{*}\right] ; L^{p}(\Omega)\right)}\left\|u^{\xi}\right\|_{C\left(\left[0, T^{*}\right] ; L^{p}(\Omega)\right)} \\
\leq & \left\|u_{0}\right\|_{p}+C(\Omega) T^{* \frac{1}{2}}\left\|u_{0}\right\|_{p}\left\|u^{\xi}\right\|_{C\left(\left[0, T^{*}\right] ; L^{p}(\Omega)\right)} \text { for each } t \in\left[0, T^{*}\right],
\end{aligned}
$$

which leads to

$$
\left\|u^{\xi}\right\|_{C\left(\left[0, T^{*}\right] ; L^{p}(\Omega)\right)} \leq \frac{1}{1-C(\Omega) T^{* \frac{1}{2}}\left\|u_{0}\right\|_{p}}\left\|u_{0}\right\|_{p} .
$$


By choosing $T^{*}:=T^{*}\left(\left\|u_{0}\right\|_{p}, \Omega\right)$ so that

$$
\frac{1}{1-C(\Omega) T^{* \frac{1}{2}}\left\|u_{0}\right\|_{p}} \leq 2
$$

we get from (2.14) that $\Psi(\xi) \in K$. Therefore, $\Psi(K) \subseteq K$. Third, we claim that $\Psi$ is a strict contraction for some $T^{*}>0$ satisfying (2.15). In fact, given $\xi_{1}, \xi_{2} \in K$, write $u_{i}=\Psi\left(\xi_{i}\right)$ and $v_{i}=v^{\xi_{i}}(i=1,2)$. Let $w:=u_{1}-u_{2}$. Then one can easily check that $w$ belongs to $C\left(\left[0, T^{*}\right] ; L^{p}(\Omega)\right)$ and satisfies

$$
\begin{cases}\partial_{t} w-\Delta w+\nabla \cdot\left(w \nabla v_{1}\right)+\nabla \cdot\left(u_{2} \nabla\left(v_{1}-v_{2}\right)\right)=0, & \text { in } \Omega \times\left(0, T^{*}\right] \\ w=0, & \text { on } \partial \Omega \times\left(0, T^{*}\right], \\ w(x, 0)=0, & \text { in } \Omega .\end{cases}
$$

Thus, we have

$$
w(t)=-\int_{0}^{t} S_{p}(t-s) \nabla \cdot\left[w \nabla v_{1}+u_{2} \nabla\left(v_{1}-v_{2}\right)\right] d s, \quad t \in\left[0, T^{*}\right] .
$$

This, along with Lemma 2.3, yields that when $t \in\left[0, T^{*}\right]$,

$$
\begin{gathered}
\|w(t)\|_{p} \leq C(\Omega) \int_{0}^{t}(t-s)^{-\frac{1}{2}}\left(\left\|\nabla v_{1}\right\|_{\left[L^{\infty}(\Omega)\right]^{n}}\|w\|_{p}+\left\|\nabla\left(v_{1}-v_{2}\right)\right\|_{\left[L^{\infty}(\Omega)\right]^{n}}\left\|u_{2}\right\|_{p}\right) d s \\
\leq C(\Omega) T^{* \frac{1}{2}}\left(\left\|\nabla v_{1}\right\|_{L^{\infty}\left(0, T^{*} ;\left[L^{\infty}(\Omega)\right]^{n}\right)}\|w\|_{C\left(\left[0, T^{*}\right] ; L^{p}(\Omega)\right)}+\left\|\nabla\left(v_{1}-v_{2}\right)\right\|_{L^{\infty}\left(0, T^{*} ;\left[L^{\infty}(\Omega)\right]^{n}\right)}\left\|u_{2}\right\|_{C\left(\left[0, T^{*}\right] ; L^{p}(\Omega)\right)}\right) .
\end{gathered}
$$

The above inequality, together with (2.8) and the fact that $\xi_{1}, u_{2} \in K$, indicates that when $t \in$ $\left[0, T^{*}\right]$,

$$
\|w(t)\|_{p} \leq C(\Omega) T^{* \frac{1}{2}}\left\|u_{0}\right\|_{p}\left(\|w\|_{C\left(\left[0, T^{*}\right] ; L^{p}(\Omega)\right)}+\left\|\xi_{1}-\xi_{2}\right\|_{C\left(\left[0, T^{*}\right] ; L^{p}(\Omega)\right)}\right),
$$

from which it follows that

$$
\left\|\Psi\left(\xi_{1}\right)-\Psi\left(\xi_{2}\right)\right\|_{C\left(\left[0, T^{*}\right] ; L^{p}(\Omega)\right)} \leq \frac{C(\Omega) T^{* \frac{1}{2}}\left\|u_{0}\right\|_{p}}{1-C(\Omega) T^{* \frac{1}{2}}\left\|u_{0}\right\|_{p}}\left\|\xi_{1}-\xi_{2}\right\|_{C\left(\left[0, T^{*}\right] ; L^{p}(\Omega)\right)} .
$$

Choosing $T^{*}:=T^{*}\left(\left\|u_{0}\right\|_{p}, \Omega\right)$ satisfying (2.15) and

$$
\frac{C(\Omega) T^{* \frac{1}{2}}\left\|u_{0}\right\|_{p}}{1-C(\Omega) T^{* \frac{1}{2}}\left\|u_{0}\right\|_{p}}<1
$$

we see from (2.16) that $\Psi$ is a strict contraction map from $K$ to $K$. Thus, according to the contraction mapping theorem, $\Psi$ has a unique fixed point $u$ in $K$.

Finally, we show (2.13). Indeed, one can easily check that $\Psi(0)=S_{p}(t) u_{0} \in K$. Then by taking $\xi_{1}=u$ and $\xi_{2}=0$ in (2.16), we have

$$
\begin{aligned}
& \|u\|_{C\left(\left[0, T^{*}\right] ; L^{p}(\Omega)\right)}=\|\Psi(u)\|_{C\left(\left[0, T^{*}\right] ; L^{p}(\Omega)\right)} \\
\leq & \|\Psi(0)\|_{C\left(\left[0, T^{*}\right] ; L^{p}(\Omega)\right)}+\|\Psi(u)-\Psi(0)\|_{C\left(\left[0, T^{*}\right] ; L^{p}(\Omega)\right)} \\
\leq & \left\|u_{0}\right\|_{p}+\frac{C(\Omega) T^{* \frac{1}{2}}\left\|u_{0}\right\|_{p}}{1-C(\Omega) T^{* \frac{1}{2}}\left\|u_{0}\right\|_{p}}\|u\|_{C\left(\left[0, T^{*}\right] ; L^{p}(\Omega)\right)},
\end{aligned}
$$


which leads to (2.13).

Step 2. We show that the solution of the system (1.1) is unique.

By contradiction, we suppose that it is not true. Then we could find another solution $(\tilde{u}, \tilde{v}) \in$ $C\left(\left[0, T^{*}\right] ; L^{p}(\Omega)\right) \times C\left(\left(0, T^{*}\right] ; W^{2, p}(\Omega) \cap W_{0}^{1, p}(\Omega)\right)$ to the system (1.1) (with the initial datum $\left.u_{0}\right)$, differing from the solution $(u, v)$ obtained in Step 1. Write

$$
\varrho:=\max \left\{\|u\|_{C\left(\left[0, T^{*}\right] ; L^{p}(\Omega)\right)},\|\tilde{u}\|_{C\left(\left[0, T^{*}\right] ; L^{p}(\Omega)\right)}\right\},
$$

and

$$
t_{0}:=\inf \left\{t \in\left[0, T^{*}\right] \mid u(t) \neq \tilde{u}(t)\right\} .
$$

It is obvious that $0 \leq t_{0}<T^{*}$. By the continuity of $u$ and $\tilde{u}$, we have $u\left(t_{0}\right)=\tilde{u}\left(t_{0}\right)$. Now, we have

$$
\begin{aligned}
u(t)-\tilde{u}(t) & =\left[S_{p}\left(t-t_{0}\right) u\left(t_{0}\right)-\int_{t_{0}}^{t} S_{p}(t-s) \nabla \cdot(u \nabla v) d s\right] \\
& -\left[S_{p}\left(t-t_{0}\right) \tilde{u}\left(t_{0}\right)-\int_{t_{0}}^{t} S_{p}(t-s) \nabla \cdot(\tilde{u} \nabla \tilde{v}) d s\right], \quad t \in\left(t_{0}, T^{*}\right] .
\end{aligned}
$$

After simple computations, we see

$$
\begin{gathered}
\|u(t)-\tilde{u}(t)\|_{p}=\left\|\int_{t_{0}}^{t} S_{p}(t-s) \nabla \cdot(u \nabla v) d s-\int_{t_{0}}^{t} S_{p}(t-s) \nabla \cdot(\tilde{u} \nabla \tilde{v}) d s\right\|_{p} \\
\leq \int_{t_{0}}^{t}\left\|S_{p}(t-s) \nabla \cdot((u-\tilde{u}) \nabla v)+S_{p}(t-s) \nabla \cdot(\tilde{u} \nabla(v-\tilde{v}))\right\|_{p} d s, \quad t \in\left(t_{0}, T^{*}\right] .
\end{gathered}
$$

This, along with Lemma 2.3, yields that for each $t \in\left(t_{0}, T^{*}\right]$,

$$
\begin{gathered}
\|u(t)-\tilde{u}(t)\|_{p} \leq C(\Omega) \int_{t_{0}}^{t}(t-s)^{-\frac{1}{2}}\left(\|(u-\tilde{u}) \nabla v\|_{p}+\|\tilde{u} \nabla(v-\tilde{v})\|_{p}\right) d s \\
\leq C(\Omega) \int_{t_{0}}^{t}(t-s)^{-\frac{1}{2}}\left(\|\nabla v\|_{\left[L^{\infty}(\Omega)\right]^{n}}\|u-\tilde{u}\|_{p}+\|\nabla(v-\tilde{v})\|_{\left[L^{\infty}(\Omega)\right]^{n}}\|\tilde{u}\|_{p}\right) d s .
\end{gathered}
$$

By the second equation of the system (1.1), Lemma 2.1, and the Sobolev embedding theorem, we have that for each $s \in\left(0, T^{*}\right]$,

$$
\|\nabla v(s)\|_{\left[L^{\infty}(\Omega)\right]^{n}} \leq C(\Omega)\|u(s)\|_{p} \quad \text { and } \quad\|\nabla(v-\tilde{v})(s)\|_{\left[L^{\infty}(\Omega)\right]^{n}} \leq C(\Omega)\|(u-\tilde{u})(s)\|_{p} .
$$

This, along with (2.19) and (2.17), implies

$$
\begin{aligned}
\|u(t)-\tilde{u}(t)\|_{p} & \leq C(\Omega) \int_{t_{0}}^{t}(t-s)^{-\frac{1}{2}}\left(\|u\|_{p}\|u-\tilde{u}\|_{p}+\|u-\tilde{u}\|_{p}\|\tilde{u}\|_{p}\right) d s \\
& \leq 4 \varrho C(\Omega)\left(t-t_{0}\right)^{\frac{1}{2}}\|u-\tilde{u}\|_{C\left(\left[t_{0}, t\right] ; L^{p}(\Omega)\right)}, \quad t \in\left(t_{0}, T^{*}\right]
\end{aligned}
$$

which leads to

$$
\|u-\tilde{u}\|_{C\left(\left[t_{0}, t\right] ; L^{p}(\Omega)\right)} \leq 4 \varrho C(\Omega)\left(t-t_{0}\right)^{\frac{1}{2}}\|u-\tilde{u}\|_{C\left(\left[t_{0}, t\right] ; L^{p}(\Omega)\right)}, \quad t \in\left(t_{0}, T^{*}\right] .
$$

Let $\epsilon>0$ satisfy that $4 \varrho C(\Omega) \epsilon^{\frac{1}{2}}<1$ and $t_{0}+\epsilon \leq T^{*}$. Then the above inequality indicates that

$$
u(s)=\tilde{u}(s), \text { when } s \in\left(t_{0}, t_{0}+\epsilon\right),
$$


which leads to a contradiction to (2.18). Hence, the desired uniqueness holds.

Step 3. We show (ii), with the aid of Lemma 2.4.

By the same argument in the proof of (2.20), we can easily check that the solution $(u, v)$ obtained in Step 1 satisfies

$$
\|v\|_{L^{\infty}\left(0, T^{*} ; L^{\infty}(\Omega)\right)}+\|\nabla v\|_{L^{\infty}\left(0, T^{*} ;\left[L^{\infty}(\Omega)\right]^{n}\right)} \leq C(\Omega)\|u\|_{C\left(\left[0, T^{*}\right] ; L^{p}(\Omega)\right)} .
$$

This, along with (2.13), implies that

$$
\|v\|_{L^{\infty}\left(0, T^{*} ; L^{\infty}(\Omega)\right)}+\|\nabla v\|_{L^{\infty}\left(0, T^{*} ;\left[L^{\infty}(\Omega)\right]^{n}\right)} \leq C\left(\left\|u_{0}\right\|_{p}, T^{*}, \Omega\right),
$$

which leads to $v \in L^{\infty}\left(0, T^{*} ; L^{\infty}(\Omega)\right)$.

Next, we define a differential operator $\mathcal{A}_{1}(t)$ by

$$
\mathcal{A}_{1}(t) \varphi:=\triangle \varphi-\nabla v \cdot \nabla \varphi-a v \varphi, \quad \varphi \in W^{2, p}(\Omega) \cap W_{0}^{1, p}(\Omega),
$$

and let $U_{1}(t, s)$ be the evolution system generated by $\mathcal{A}_{1}(t)\left(T^{*} \geq t \geq 0\right)$. Since (1.1) can be rewritten as:

$$
\begin{cases}\partial_{t} u-\Delta u+\nabla v \cdot \nabla u+a v u=b u^{2}, & \text { in } \Omega \times\left(0, T^{*}\right], \\ -\triangle v+a v-b u=0, & \text { in } \Omega \times\left(0, T^{*}\right], \\ u=0, v=0, & \text { on } \partial \Omega \times\left(0, T^{*}\right], \\ u(x, 0)=u_{0}, & \text { in } \Omega,\end{cases}
$$

we see from the variation of constants formula that

$$
u(t)=U_{1}(t, 0) u_{0}+b \int_{0}^{t} U_{1}(t, s) u^{2} d s, \quad t \in\left[0, T^{*}\right] .
$$

Then, it follows from (2.22) and Lemma 2.4 that

$$
\begin{gathered}
\|u(t)\|_{\infty} \leq\left\|U_{1}(t, 0) u_{0}\right\|_{\infty}+\left\|b \int_{0}^{t} U_{1}(t, s) u^{2}(s) d s\right\|_{\infty} \\
\leq e^{\varpi(1+t)} t^{-\frac{n}{2 p}}\left\|u_{0}\right\|_{p}+b \int_{0}^{t}(t-s)^{-\frac{n}{p}} e^{\varpi[1+(t-s)]}\left\|u^{2}(s)\right\|_{\frac{p}{2}} d s, \quad t \in\left(0, T^{*}\right],
\end{gathered}
$$

where $\varpi$ is a positive number depending on $C\left(\left\|u_{0}\right\|_{p}, T^{*}, n, \Omega\right)$. After some computations, we obtain

$$
\begin{aligned}
& \|u(t)\|_{\infty} \leq e^{\varpi(1+t)} t^{-\frac{n}{2 p}}\left\|u_{0}\right\|_{p}+b \int_{0}^{t}(t-s)^{-\frac{n}{p}} e^{\varpi[1+(t-s)]}\|u(s)\|_{p}^{2} d s \\
\leq & e^{\varpi\left(1+T^{*}\right)}\left(t^{-\frac{n}{2 p}}\left\|u_{0}\right\|_{p}+\frac{b p}{p-n} t^{1-\frac{n}{p}}\|u\|_{C\left(\left[0, T^{*}\right] ; L^{p}(\Omega)\right)}^{2}\right), \quad t \in\left(0, T^{*}\right] .
\end{aligned}
$$

Since $p>n$, the above inequality leads to $t^{\frac{n}{2 p}} u \in L^{\infty}\left(0, T^{*} ; L^{\infty}(\Omega)\right)$. In summary, we conclude that $\left.\left(t^{\frac{n}{2 p}} u, v\right) \in L^{\infty}\left(0, T^{*} ; L^{\infty}(\Omega)\right)\right]^{2}$.

Step 4. We show (iii).

Let $u_{0} \in L^{\infty}(\Omega)$. By Step 1 and Step 3, we already have $v \in L^{\infty}\left(0, T^{*} ; L^{\infty}(\Omega)\right)$ and $u \in$ $C\left(\left[0, T^{*}\right] ; L^{p}(\Omega)\right)(n<p<+\infty)$. The remainder is to show $u \in L^{\infty}\left(0, T^{*} ; L^{\infty}(\Omega)\right)$. To this end, we see from (2.22) and Lemma 2.4 that

$$
\|u(t)\|_{\infty} \leq\left\|U_{1}(t, 0) u_{0}\right\|_{\infty}+\left\|b \int_{0}^{t} U_{1}(t, s) u^{2}(s) d s\right\|_{\infty}
$$




$$
\begin{aligned}
& \leq e^{\varpi(1+t)}\left\|u_{0}\right\|_{\infty}+b \int_{0}^{t}(t-s)^{-\frac{n}{p}} e^{\varpi[1+(t-s)]}\|u(s)\|_{p}^{2} d s \\
& \leq e^{\varpi\left(1+T^{*}\right)}\left(\left\|u_{0}\right\|_{\infty}+\frac{b p}{p-n} t^{1-\frac{n}{p}}\left(\|u\|_{C\left(\left[0, T^{*}\right] ; L^{p}(\Omega)\right)}^{2}\right)<+\infty, \quad t \in\left[0, T^{*}\right],\right.
\end{aligned}
$$

where $\varpi$ is a positive number depending on $C\left(\left\|u_{0}\right\|_{\infty}, T^{*}, n, \Omega\right)$. From (2.23) and $p>n$, it follows that $u \in L^{\infty}\left(0, T^{*} ; L^{\infty}(\Omega)\right)$.

Hence, we complete the proof of Theorem 2.1.

Remark 2.3. (i) It follows from (2.13) and (2.23) that when $u_{0} \in L^{\infty}(\Omega)$,

$$
\|u\|_{L^{\infty}\left(0, T^{*} ; L^{\infty}(\Omega)\right)} \leq C\left(\left\|u_{0}\right\|_{\infty}, T^{*}, n, \Omega\right) .
$$

(ii) Let $u_{0} \in L^{\infty}(\Omega)$. Suppose that $(u, v)$ is the solution to the system (1.1) over $[0, T]$ for some $T>0$. First, by the conclusion (iii) in Theorem [2.1, we have that $(u, v) \in\left[L^{\infty}\left(0, T ; L^{\infty}(\Omega)\right)\right]^{2}$, and it follows from (2.21) that

$$
\|v\|_{L^{\infty}\left(0, T ; L^{\infty}(\Omega)\right)} \leq C M \text { and }\|\nabla v\|_{L^{\infty}\left(0, T ;\left[L^{\infty}(\Omega)\right]^{n}\right)} \leq C M
$$

where $C>0$ depends only on $\Omega$ and where

$$
M:=M\left(\left\|u_{0}\right\|_{\infty}, T\right):=\max \left\{1,\|u\|_{L^{\infty}\left(0, T ; L^{\infty}(\Omega)\right)}\right\} .
$$

Second, for each $p \in(n,+\infty)$, the above solution $(u, v) \in C\left([0, T] ; L^{p}(\Omega)\right) \times C\left((0, T] ; W^{2, p}(\Omega) \cap\right.$ $\left.W_{0}^{1, p}(\Omega)\right)$.

(iii) By (1.1) and the uniqueness given in Theorem 2.1, we can easily verify what follows: $u_{0}=0$ over $\Omega$ if and only if $u=v=0$ over $\Omega \times(0,+\infty)$.

Next, we present some estimates for the system (1.1).

Theorem 2.2. Let $u_{0} \in L^{\infty}(\Omega)$. Suppose that $(u, v)$ is the solution to the system (1.1) over $[0, T]$ for some $T>0$. Then,

$$
\begin{gathered}
\|u(\cdot, t)\|_{2} \leq e^{L_{1} M^{2} t}\left\|u_{0}\right\|_{2}, \quad t \in[0, T] \\
\|\nabla u(\cdot, t)\|_{2} \leq \frac{e^{L_{1} M^{2} t}}{\sqrt{t}}\left\|u_{0}\right\|_{2}, \quad t \in(0, T],
\end{gathered}
$$

where $L_{1}>0$ is a constant depending only on $\Omega$ and where $M$ is given by (2.25).

Proof. Multiplying the first equation of (1.1) by $e^{-l t} \partial_{t} u$, where $l>0$ will be determined later, and then integrating it over $\Omega$, we obtain

$$
\int_{\Omega}\left[\partial_{t} u-\triangle u+\nabla \cdot(u \nabla v)\right] \cdot e^{-l t} \partial_{t} u d x=0, t \in[0, T] .
$$

From the above, the second equation of the system (1.1), (2.24), Cauchy's inequality, and Lemma 2.1, we find

$$
e^{-l t} \int_{\Omega}\left|\partial_{t} u\right|^{2} d x+\frac{1}{2} \frac{d}{d t}\left(e^{-l t} \int_{\Omega}|\nabla u|^{2} d x\right)
$$




$$
\begin{aligned}
& =-e^{-l t} \int_{\Omega}\left(\nabla u \cdot \nabla v+a u v-b u^{2}\right) \cdot \partial_{t} u d x-\frac{l}{2} e^{-l t} \int_{\Omega}|\nabla u|^{2} d x \\
& \leq \frac{1}{2} e^{-l t} \int_{\Omega}\left|\partial_{t} u\right|^{2} d x+C(\Omega) M^{2} e^{-l t} \int_{\Omega}|\nabla u|^{2} d x \\
& +C(\Omega) M^{2} e^{-l t} \int_{\Omega}|u|^{2} d x+\frac{3 b^{2}}{2} M^{2} e^{-l t} \int_{\Omega}|u|^{2} d x-\frac{l}{2} e^{-l t} \int_{\Omega}|\nabla u|^{2} d x, t \in[0, T],
\end{aligned}
$$

which yields that when $t \in[0, T]$,

$$
\begin{aligned}
& \frac{1}{2} e^{-l t} \int_{\Omega}\left|\partial_{t} u\right|^{2} d x+\frac{1}{2} \frac{d}{d t}\left(e^{-l t} \int_{\Omega}|\nabla u|^{2} d x\right) \\
\leq & C(\Omega) M^{2} e^{-l t} \int_{\Omega}|\nabla u|^{2} d x+\left(C(\Omega)+\frac{3 b^{2}}{2}\right) M^{2} e^{-l t} \int_{\Omega}|u|^{2} d x-\frac{l}{2} e^{-l t} \int_{\Omega}|\nabla u|^{2} d x .
\end{aligned}
$$

Meanwhile, multiplying the first equation of (1.1) by $e^{-l t} u$, and integrating it over $\Omega$, we see

$$
\int_{\Omega}\left[\partial_{t} u-\triangle u+\nabla \cdot(u \nabla v)\right] \cdot e^{-l t} u d x=0, t \in[0, T] .
$$

From the above, the second equation of the system (1.1), (2.24), Cauchy's inequality, and Lemma 2.1, we obtain

$$
\begin{aligned}
& \frac{1}{2} \frac{d}{d t}\left(e^{-l t} \int_{\Omega}|u|^{2} d x\right)+e^{-l t} \int_{\Omega}|\nabla u|^{2} d x \\
= & -e^{-l t} \int_{\Omega}(\nabla u \cdot \nabla v+a v-b u) u d x-\frac{l}{2} e^{-l t} \int_{\Omega}|u|^{2} d x \\
\leq & \frac{1}{2} e^{-l t} \int_{\Omega}|\nabla u|^{2} d x+C(\Omega) M^{2} e^{-l t} \int_{\Omega}|u|^{2} d x+\frac{a}{2} e^{-l t} \int_{\Omega}|v|^{2} d x \\
+ & \left(\frac{a}{2}+b\right) e^{-l t} \int_{\Omega}|u|^{2} d x-\frac{l}{2} e^{-l t} \int_{\Omega}|u|^{2} d x, t \in[0, T],
\end{aligned}
$$

from which, it follows that

$$
\begin{aligned}
& \frac{d}{d t}\left(\frac{1}{2} e^{-l t} \int_{\Omega}|u|^{2} d x\right)+\frac{1}{2} e^{-l t} \int_{\Omega}|\nabla u|^{2} d x \\
\leq & (C(\Omega)+a+b) M^{2} e^{-l t} \int_{\Omega}|u|^{2} d x-\frac{l}{2} e^{-l t} \int_{\Omega}|u|^{2} d x, t \in[0, T] .
\end{aligned}
$$

According to (2.28), (2.29), and Poincaré inequality, there is a constant $l=L_{1} M^{2}>0$, where $L_{1}>0$ is a constant depending only on $\Omega$, so that

$$
\begin{aligned}
& \frac{d}{d t}\left(e^{-L_{1} M^{2} t} \int_{\Omega}|\nabla u(x, t)|^{2} d x\right)+e^{-L_{1} M^{2} t} \int_{\Omega}\left|\partial_{t} u(x, t)\right|^{2} d x \leq 0, t \in[0, T] \\
& \frac{d}{d t}\left(e^{-L_{1} M^{2} t} \int_{\Omega}|u(x, t)|^{2} d x\right)+e^{-L_{1} M^{2} t} \int_{\Omega}|\nabla u(x, t)|^{2} d x \leq 0, t \in[0, T] .
\end{aligned}
$$

We now show (2.26). Integrating (2.31) over $(0, t)$, we obtain

$$
e^{-L_{1} M^{2} t} \int_{\Omega}|u(x, t)|^{2} d x+\int_{0}^{t}\left(e^{-L_{1} M^{2} s} \int_{\Omega}|\nabla u(x, s)|^{2} d x\right) d s \leq\left\|u_{0}\right\|_{2}^{2}, t \in[0, T],
$$

which leads to (2.26). By (2.30), we see that the function $t \rightarrow e^{-L_{1} M^{2} t} \int_{\Omega}|\nabla u(x, t)|^{2} d x$ is nonincreasing over $[0, T]$. Thus,

$$
t e^{-L_{1} M^{2} t} \int_{\Omega}|\nabla u(x, t)|^{2} d x \leq \int_{0}^{t}\left(e^{-L_{1} M^{2} s} \int_{\Omega}|\nabla u(x, s)|^{2} d x\right) d s, t \in[0, T] .
$$

Finally, (2.27) follows from (2.33) and (2.32) at once. This completes the proof. 


\section{Local interpolation inequality for the system (1.1)}

First of all, since $\Omega \subset \mathbb{R}^{n}$ is a bounded domain with a $C^{2}$ boundary $\partial \Omega$, it follows from Theorem 8 in [3] that for each $g \in \partial \Omega$, there is a triplet $\left(x_{g}, R_{g}, \delta_{g}\right) \in \Omega \times \mathbb{R}^{+} \times(0,1]$ such that

$$
\left|g-x_{g}\right|<R_{g} \text { and } \Omega \cap B_{\left(1+2 \delta_{g}\right) R_{g}} \text { is star-shaped with } x_{g}
$$

where $B_{\left(1+2 \delta_{g}\right) R_{g}}$ stands for the open ball centered at $x_{g}$ and of radius $\left(1+2 \delta_{g}\right) R_{g}$.

Throughout this section, we arbitrarily fix $u_{0} \in L^{\infty}(\Omega)$ and $T>0$ so that the system (1.1) has a unique solution $(u, v)$ over $[0, T]$; we arbitrarily fix $g \in \partial \Omega$ with $\left(x_{g}, R_{g}, \delta_{g}\right) \in \Omega \times \mathbb{R}^{+} \times(0,1]$ satisfying (3.1); we simply write $B_{R}$ for the open ball centered at $x_{g}$ and of radius $R$.

The main result of this section is the following Theorem 3.1, which builds up a local interpolation inequality for the solution $(u, v)$.

Theorem 3.1. For each $r \in\left(0, R_{g}\right)$ with $B_{r}:=B\left(x_{g}, r\right) \subset \Omega$, there are two constants $D>0$ and $\gamma \in(0,1)$, which depend only on $\Omega, r, R_{g}, \delta_{g}, T$, and $M$ (with $M$ given by (2.25)) so that

$$
\int_{\Omega \cap B_{R_{g}}}|u(x, T)|^{2} d x \leq\left(D \int_{\Omega}\left|u_{0}(x)\right|^{2} d x\right)^{\gamma}\left(2 \int_{B_{r}}|u(x, T)|^{2} d x\right)^{1-\gamma} .
$$

To prove Theorem 3.1, we need several lemmas. We start with introducing two functions in the following manner: Arbitrarily take $z \in H^{1}\left(0, T ; L^{2}\left(\Omega \cap B_{\left(1+2 \delta_{g}\right) R_{g}}\right)\right) \cap L^{2}\left(0, T ; H^{2} \cap H_{0}^{1}(\Omega \cap\right.$ $\left.\left.B_{\left(1+2 \delta_{g}\right) R_{g}}\right)\right)$, then for each $\lambda>0$, we define functions

$$
G_{\lambda}(x, t):=\frac{1}{(T-t+\lambda)^{n / 2}} e^{-\frac{|x-x|^{2}}{4(T-t+\lambda)}}, \quad(x, t) \in \mathbb{R}^{n} \times[0, T]
$$

and

$$
N_{\lambda}(t):=\frac{\int_{\Omega \cap B_{\left(1+2 \delta_{g}\right) R_{g}}}|\nabla z(x, t)|^{2} G_{\lambda}(x, t) d x}{\int_{\Omega \cap B_{\left(1+2 \delta_{g}\right) R_{g}}}|z(x, t)|^{2} G_{\lambda}(x, t) d x}
$$

when $t \in(0, T]$ and $\int_{\Omega \cap B_{\left(1+2 \delta_{g}\right) R_{g}}}|z(x, t)|^{2} G_{\lambda}(x, t) d x \neq 0$.

Remark 3.1. The above $N_{\lambda}(\cdot)$ can be viewed as a localized frequency function. We simply call it the frequency function.

Lemma 3.1. The frequency function $N_{\lambda}(\cdot)$ (given by (3.4)) has the following properties:

(i) When $t \in(0, T], \lambda>0$, and $\int_{\Omega \cap B_{\left(1+2 \delta_{g}\right) R_{g}}}|z(x, t)|^{2} G_{\lambda}(x, t) d x \neq 0$,

$$
\begin{aligned}
& \frac{1}{2} \frac{d}{d t} \int_{\Omega \cap B_{\left(1+2 \delta_{g}\right) R_{g}}}|z(x, t)|^{2} G_{\lambda}(x, t) d x+N_{\lambda}(t) \int_{\Omega \cap B_{\left(1+2 \delta_{g}\right) R_{g}}}|z(x, t)|^{2} G_{\lambda}(x, t) d x \\
= & \int_{\Omega \cap B_{\left(1+2 \delta_{g}\right) R_{g}}} z(x, t)\left(\partial_{t} z(x, t)-\triangle z(x, t)\right) G_{\lambda}(x, t) d x .
\end{aligned}
$$

(ii) When $t \in(0, T], \lambda>0$, and $\int_{\Omega \cap B_{\left(1+2 \delta_{g}\right) R_{g}}}|z(x, t)|^{2} G_{\lambda}(x, t) d x \neq 0$,

$$
\frac{d}{d t} N_{\lambda}(t) \leq \frac{1}{T-t+\lambda} N_{\lambda}(t)+\frac{\int_{\Omega \cap B_{\left(1+2 \delta_{g}\right) R_{g}}}\left(\partial_{t} z(x, t)-\triangle z(x, t)\right)^{2} G_{\lambda}(x, t) d x}{\int_{\Omega \cap B_{\left(1+2 \delta_{g}\right) R_{g}}}|z(x, t)|^{2} G_{\lambda}(x, t) d x}
$$


Proof. The proof can be done by the same method used in Lemma 3.1 of [23] (see also Lemma 2.3 in [24]). We omit the details.

Lemma 3.2. Suppose that $\int_{\Omega \cap B_{R_{g}}}|u(x, T)|^{2} d x \neq 0$. Then there are positive constants $L_{2}, L_{3}, L_{4}$, and $L_{5}$ (depending only on $\Omega, R_{g}$, and $\delta_{g}$ ) so that

$$
0<\frac{\mathbb{E}}{\int_{\Omega \cap B_{\left(1+\delta_{g}\right) R_{g}}}|u(x, t)|^{2} d x} \leq e^{\frac{L_{2}}{\theta}}, \text { when } T-\theta \leq t \leq T,
$$

where

$$
\frac{1}{\theta}:=L_{3} \ln \left(e^{L_{4} M^{2} T+L_{5}\left(1+\frac{1}{T}\right)} \frac{\mathbb{E}}{\int_{\Omega \cap B_{R_{g}}}|u(x, T)|^{2} d x}\right) \in\left(\frac{1}{\min \{1, T / 2\}},+\infty\right),
$$

with $M$ given by (2.25) and

$$
\mathbb{E}:=\int_{\Omega}|u(x, 0)|^{2} d x+\int_{0}^{T} \int_{\Omega}|u(x, s)|^{2} d x d s .
$$

Proof. Write $R_{1}:=\left(1+\delta_{g}\right) R_{g}$. Let $\sigma \in C_{0}^{\infty}\left(\mathbb{R}^{n}\right)$ be such that

$$
\operatorname{supp} \sigma \subset B_{R_{1}}, 0 \leq \sigma \leq 1 \text {, and } \sigma=1 \text { on } B_{\left(1+\delta_{g} / 2\right) R_{g}} \text {. }
$$

Then, there is $C=C\left(R_{g}, \delta_{g}\right)>0$ so that

$$
\begin{aligned}
& |\nabla \sigma(x)| \leq C\left(R_{g}, \delta_{g}\right), x \in \mathbb{R}^{n} \\
& \nabla \sigma(x)=0, \quad x \in B_{\left(1+\delta_{g} / 2\right) R_{g}} .
\end{aligned}
$$

Multiplying the first equation of (1.1) by $e^{-\frac{\left|x-x_{g}\right|^{2}}{h}} \sigma^{2} u$, where $h>0$ will be determined later, integrating it over $\Omega \cap B_{R_{1}}$, and then using the integration by parts, we find

$$
\begin{aligned}
& \frac{1}{2} \frac{d}{d t} \int_{\Omega \cap B_{R_{1}}}|\sigma u|^{2} \cdot e^{-\frac{\left|x-x_{g}\right|^{2}}{h}} d x+\int_{\Omega \cap B_{R_{1}}} \nabla u \cdot \nabla\left(e^{-\frac{\left|x-x_{g}\right|^{2}}{h}} \sigma^{2} u\right) d x \\
= & \int_{\Omega \cap B_{R_{1}}} u \nabla v \cdot \nabla\left(e^{-\frac{|x-x g|^{2}}{h}} \sigma^{2} u\right) d x, \quad t \in[0, T] .
\end{aligned}
$$

Meanwhile, one can directly check

$$
\nabla\left(e^{-\frac{\left|x-x_{g}\right|^{2}}{h}} \sigma^{2} u\right)=e^{-\frac{\left|x-x_{g}\right|^{2}}{h}} \sigma^{2} \nabla u+2 e^{-\frac{\left|x-x_{g}\right|^{2}}{h}} u \sigma \nabla \sigma-2 \sigma^{2} u e^{-\frac{\left|x-x_{g}\right|^{2}}{h}} \frac{x-x_{g}}{h}, \quad x \in \Omega, t \in[0, T] .
$$

These lead to that when $t \in[0, T]$,

$$
\begin{aligned}
& \frac{1}{2} \frac{d}{d t} \int_{\Omega \cap B_{R_{1}}}|\sigma u|^{2} \cdot e^{-\frac{\left|x-x_{g}\right|^{2}}{h}} d x+\int_{\Omega \cap B_{R_{1}}} e^{-\frac{\left|x-x_{g}\right|^{2}}{h}} \sigma^{2}|\nabla u|^{2} d x \\
= & \int_{\Omega \cap B_{R_{1}}} e^{-\frac{\left|x-x_{g}\right|^{2}}{h}} \sigma^{2} u \nabla u \cdot \nabla v d x+\int_{\Omega \cap B_{R_{1}}} 2 e^{-\frac{\left|x-x_{g}\right|^{2}}{h}} \sigma u^{2} \nabla \sigma \cdot \nabla v d x \\
- & \int_{\Omega \cap B_{R_{1}}} 2 e^{-\frac{\left|x-x_{g}\right|^{2}}{h}} \sigma^{2} u^{2} \cdot \frac{x-x_{g}}{h} \cdot \nabla v d x-\int_{\Omega \cap B_{R_{1}}} 2 e^{-\frac{\left|x-x_{g}\right|^{2}}{h}} u \sigma \nabla \sigma \cdot \nabla u d x
\end{aligned}
$$




$$
+\int_{\Omega \cap B_{R_{1}}} 2 e^{-\frac{\left|x-x_{g}\right|^{2}}{h}} \sigma^{2} u \frac{x-x_{g}}{h} \cdot \nabla u d x .
$$

The above equation, along with (2.24), yields that when $t \in[0, T]$,

$$
\begin{aligned}
& \frac{1}{2} \frac{d}{d t} \int_{\Omega \cap B_{R_{1}}}|\sigma u|^{2} \cdot e^{-\frac{\left|x-x_{g}\right|^{2}}{h}} d x+\int_{\Omega \cap B_{R_{1}}} e^{-\frac{\left|x-x_{g}\right|^{2}}{h}} \sigma^{2}|\nabla u|^{2} d x \\
\leq & \int_{\Omega \cap B_{R_{1}}} C_{1} M e^{-\frac{\left|x-x_{g}\right|^{2}}{h}} \sigma^{2}|u| \cdot|\nabla u| d x+C_{1} M \int_{\Omega \cap B_{R_{1}}}|\sigma||\nabla \sigma| e^{-\frac{\left|x-x_{g}\right|^{2}}{h}}|u|^{2} d x \\
+ & C_{1} M \cdot \frac{R_{1}}{h} \int_{\Omega \cap B_{R_{1}}} e^{-\frac{\left|x-x_{g}\right|^{2}}{h}} \sigma^{2}|u|^{2} d x+\int_{\Omega \cap B_{R_{1}}} 2|\sigma||\nabla \sigma| e^{-\frac{\left|x-x_{g}\right|^{2}}{h}}|u| \cdot|\nabla u| d x \\
+ & \int_{\Omega \cap B_{R_{1}}} 2 \frac{R_{1}}{h} e^{-\frac{\left|x-x_{g}\right|^{2}}{h}} \sigma^{2}|u| \cdot|\nabla u| d x,
\end{aligned}
$$

where $C_{1}$ is a positive constant depending only on $\Omega$. Using Cauchy's inequality in the above inequality gives

$$
\begin{aligned}
& \frac{1}{2} \frac{d}{d t} \int_{\Omega \cap B_{R_{1}}}|\sigma u|^{2} \cdot e^{-\frac{\left|x-x_{g}\right|^{2}}{h}} d x+\int_{\Omega \cap B_{R_{1}}} e^{-\frac{\left|x-x_{g}\right|^{2}}{h}}|\sigma \nabla u|^{2} d x \\
\leq & \frac{1}{2} \int_{\Omega \cap B_{R_{1}}} e^{-\frac{\left|x-x_{g}\right|^{2}}{h}}|\sigma \nabla u|^{2} d x+8 \int_{\Omega \cap B_{R_{1}}} e^{-\frac{\left|x-x_{g}\right|^{2}}{h}}|\nabla \sigma|^{2}|u|^{2} d x \\
+ & C_{1}\left(M^{2}+\frac{R_{1}^{2}}{h^{2}}\right) \int_{\Omega \cap B_{R_{1}}}|\sigma u|^{2} \cdot e^{-\frac{\left|x-x_{g}\right|^{2}}{h}} d x, t \in[0, T] .
\end{aligned}
$$

Moving the term $\frac{1}{2} \int_{\Omega \cap B_{R_{1}}} e^{-\frac{\left|x-x_{g}\right|^{2}}{h}}|\sigma \nabla u|^{2} d x$ to the left hand side in the above, using (3.9) and (3.10), we deduce that when $t \in[0, T]$,

$$
\begin{aligned}
& \frac{d}{d t} \int_{\Omega \cap B_{R_{1}}}|\sigma u|^{2} \cdot e^{-\frac{\left|x-x_{g}\right|^{2}}{h}} d x \leq C_{2} \int_{\Omega \cap\left(B_{R_{1}} \backslash B_{\left(1+\delta_{g} / 2\right) R_{g}}\right)} e^{-\frac{\left|x-x_{g}\right|^{2}}{h}}|u|^{2} d x \\
+ & C_{1}\left(M^{2}+\frac{R_{1}^{2}}{h^{2}}\right) \int_{\Omega \cap B_{R_{1}}}|\sigma u|^{2} \cdot e^{-\frac{\left|x-x_{g}\right|^{2}}{h}} d x, \\
\leq & C_{2} e^{-\frac{\left(1+\delta_{g} / 2\right)^{2} R_{g}^{2}}{h}} \int_{\Omega \cap B_{R_{1}}}|u|^{2} d x+C_{1}\left(M^{2}+\frac{R_{1}^{2}}{h^{2}}\right) \int_{\Omega \cap B_{R_{1}}}|\sigma u|^{2} \cdot e^{-\frac{\left|x-x_{g}\right|^{2}}{h}} d x,
\end{aligned}
$$

where $C_{2}>1$ is a constant depending only on $R_{g}$ and $\delta_{g}$. Multiplying the above inequality by $\exp \left(-C_{1}\left(M^{2}+\frac{R_{1}^{2}}{h^{2}}\right) t\right)$ and then integrating it between $t$ and $T$, we obtain

$$
\begin{aligned}
& \int_{\Omega \cap B_{R_{1}}}|\sigma u(x, T)|^{2} \cdot e^{-\frac{\left|x-x_{g}\right|^{2}}{h}} d x \\
\leq & \exp \left(C_{1}\left(M^{2}+\frac{R_{1}^{2}}{h^{2}}\right)(T-t)\right) \int_{\Omega \cap B_{R_{1}}}|\sigma u(x, t)|^{2} \cdot e^{-\frac{|x-x g|^{2}}{h}} d x \\
+ & C_{2} e^{-\frac{\left(1+\delta_{g} / 2\right)^{2} R_{g}^{2}}{h}} \exp \left(C_{1}\left(M^{2}+\frac{R_{1}^{2}}{h^{2}}\right)(T-t)\right) \int_{t}^{T} \int_{\Omega \cap B_{R_{1}}}|u(x, s)|^{2} d x d s, \quad t \in[0, T],
\end{aligned}
$$

from which and (3.8), it follows that when $t \in[0, T]$,

$$
\int_{\Omega \cap B_{R_{g}}}|u(x, T)|^{2} d x \leq e^{\frac{R_{g}^{2}}{h}} \int_{\Omega \cap B_{R_{g}}}|\sigma u(x, T)|^{2} \cdot e^{-\frac{\left|x-x_{g}\right|^{2}}{h}} d x
$$




$$
\begin{aligned}
& \leq e^{\frac{R_{g}^{2}}{h}} \exp \left(C_{1}\left(M^{2}+\frac{R_{1}^{2}}{h^{2}}\right)(T-t)\right) \int_{\Omega \cap B_{R_{1}}}|u(x, t)|^{2} d x \\
& +C_{2} e^{\frac{-\left(1+\delta_{g} / 2\right)^{2} R_{g}^{2}+R_{g}^{2}}{h}} \exp \left(C_{1}\left(M^{2}+\frac{R_{1}^{2}}{h^{2}}\right)(T-t)\right) \int_{t}^{T} \int_{\Omega \cap B_{R_{1}}}|u(x, s)|^{2} d x d s .
\end{aligned}
$$

Next, we let

$$
l:=\frac{\delta_{g}+\delta_{g}^{2} / 4}{2 C_{1}\left(1+\delta_{g}\right)^{2}}
$$

Then we have

$$
\frac{C_{1} l R_{1}^{2}}{h}=\frac{\left(1+\delta_{g} / 2\right)^{2} R_{g}^{2}-R_{g}^{2}}{2 h} .
$$

Choosing $h$ sufficiently small so that $0<l h<\min \left\{1, \frac{T}{2}\right\}$, and then using (3.11), we see that when $\frac{T}{2}<T-l h \leq t \leq T$

$$
\begin{aligned}
& \int_{\Omega \cap B_{R_{g}}}|u(x, T)|^{2} d x \leq e^{\frac{R_{g}^{2}}{h}} \exp \left(C_{1}\left(M^{2}+\frac{R_{1}^{2}}{h^{2}}\right) l h\right) \int_{\Omega \cap B_{R_{1}}}|u(x, t)|^{2} d x \\
+ & C_{2} e^{\frac{-\left(1+\delta_{g} / 2\right)^{2} R_{g}^{2}+R_{g}^{2}}{h}} \exp \left(C_{1}\left(M^{2}+\frac{R_{1}^{2}}{h^{2}}\right) l h\right) \int_{t}^{T} \int_{\Omega \cap B_{R_{1}}}|u(x, s)|^{2} d x d s
\end{aligned}
$$

This, together with (3.13) and (3.7), shows that when $\frac{T}{2}<T-l h \leq t \leq T$,

$$
\begin{aligned}
\int_{\Omega \cap B_{R_{g}}}|u(x, T)|^{2} d x & \leq e^{\frac{\left(1+\delta_{g} / 2\right)^{2} R_{g}^{2}+R_{g}^{2}}{2 h}} \exp \left(C_{1} M^{2} l h\right) \int_{\Omega \cap B_{R_{1}}}|u(x, t)|^{2} d x \\
& +C_{2} e^{\frac{-\left(1+\delta_{g} / 2\right)^{2} R_{g}^{2}+R_{g}^{2}}{2 h}} \exp \left(C_{1} M^{2} l h\right) \mathbb{E} .
\end{aligned}
$$

Meanwhile, since $\int_{\Omega \cap B_{R_{g}}}|u(x, T)|^{2} d x \neq 0$, we have $u(\cdot, T) \neq 0$. This, along with the continuity of $u$ and (3.7), shows that

$$
\mathbb{E}>0 \text {. }
$$

Now, we take

$$
h:=\frac{R_{g}^{2}\left(\delta_{g}+\delta_{g}^{2} / 4\right) / 2}{\ln \left(\mathcal{K} \frac{C_{2} \exp \left(\left(C_{1}+L_{1}\right) M^{2} T\right) \mathbb{E}}{\frac{1}{e} \int_{\Omega \cap B_{R_{g}}}|u(x, T)|^{2} d x}\right)},
$$

where $\mathcal{K}=e^{\left(R_{g}^{2}\left(\delta_{g}+\delta_{g}^{2} / 4\right) / 2\right)\left(\frac{2}{T}+1\right) l}$ and $L_{1}$ is given by Theorem 2.2. One can directly check from (3.16), (3.7), (2.26), and $C_{2}>1$ that $0<l h<\min \left\{1, \frac{T}{2}\right\}$ and

$$
C_{2} e^{\frac{-\left(1+\delta_{g} / 2\right)^{2} R_{g}^{2}+R_{g}^{2}}{2 h}} \exp \left(C_{1} M^{2} T\right) \mathbb{E} \leq \frac{1}{e} \int_{\Omega \cap B_{R_{g}}}|u(x, T)|^{2} d x .
$$

Then by (3.17), and (3.14), we find that when $\frac{T}{2}<T-l h \leq t \leq T$,

$$
\left(1-\frac{1}{e}\right) \int_{\Omega \cap B_{R_{g}}}|u(x, T)|^{2} d x \leq e^{\frac{\left(1+\delta_{g} / 2\right)^{2} R_{g}^{2}+R_{g}^{2}}{2 h}} \exp \left(C_{1} M^{2} T\right) \int_{\Omega \cap B_{R_{1}}}|u(x, t)|^{2} d x
$$


which, along with (3.15), (3.17), and $C_{2}>1$, leads to

$$
0<\mathbb{E} \leq e^{\frac{\left(1+\delta_{g} / 2\right)^{2} R_{g}^{2}}{h}} \int_{\Omega \cap B_{R_{1}}}|u(x, t)|^{2} d x, \quad \frac{T}{2}<T-l h \leq t \leq T .
$$

Let $\theta:=l h$. Then it follows from (3.12), (3.16), and (3.18) that when $\frac{T}{2}<T-\theta \leq t \leq T$,

$$
\begin{gathered}
0<\frac{\mathbb{E}}{\int_{\Omega \cap B_{R_{1}}}|u(x, t)|^{2} d x} \leq e^{\frac{\left(1+\delta_{g} / 2\right)^{2} R_{g}^{2}}{h}}=e^{\frac{\left(\delta_{g}+\delta_{g}^{2} / 4\right)\left(1+\delta_{g} / 2\right)^{2} R_{g}^{2}}{2 C_{1}\left(1+\delta_{g}\right)^{2}} \frac{1}{\theta}} ; \\
\frac{1}{\theta}=\frac{4 C_{1}\left(1+\delta_{g}\right)^{2}}{R_{g}^{2}\left(\delta_{g}+\delta_{g}^{2} / 4\right)^{2}} \ln \left(e C_{2} \exp \left(\left(C_{1}+L_{1}\right) M^{2} T\right) e^{\left(\frac{2}{T}+1\right) \frac{R_{g}^{2}\left(\delta_{g}+\delta_{g}^{2} / 2\right)^{2}}{4 C_{1}\left(1+\delta_{g}\right)^{2}}} \frac{\mathbb{E}}{\int_{\Omega \cap B_{R_{g}}}|u(x, T)|^{2} d x}\right) .
\end{gathered}
$$

These lead to (3.5) and (3.6). Thus we complete the proof.

Remark 3.2. Lemma 3.2 implies that if $\int_{\Omega \cap B_{R_{g}}}|u(x, T)|^{2} d x \neq 0$, then $\int_{\Omega^{\prime} B_{\left(1+\delta_{g}\right) R_{g}}}|u(x, t)|^{2} d x \neq 0$, for any $T-\theta \leq t \leq T$, where $\theta$ is given by (3.6).

The following lemma is quoted from [8, 24] (see, for instance, (2.3.15) on page 691 in [24]).

Lemma 3.3. Let $G$ be a bounded domain in $\mathbb{R}^{n}$ with a $C^{2}$ boundary $\partial G$. Let $x_{0} \in G$. Then, for each $f \in H_{0}^{1}(G)$ and each $\lambda>0$,

$$
\int_{G} \frac{\left|x-x_{0}\right|^{2}}{8 \lambda}|f(x)|^{2} e^{-\frac{\left|x-x_{0}\right|^{2}}{4 \lambda}} d x \leq 2 \lambda \int_{G}|\nabla f(x)|^{2} e^{-\frac{\left|x-x_{0}\right|^{2}}{4 \lambda}} d x+\frac{n}{2} \int_{G}|f(x)|^{2} e^{-\frac{\left|x-x_{0}\right|^{2}}{4 \lambda}} d x .
$$

Now, we are in the position to prove Theorem 3.1

Proof of Theorem 3.1. Arbitrarily fix $r \in\left(0, R_{g}\right)$ with $B_{r}:=B\left(x_{g}, r\right) \subset \Omega$. Without loss of generality, we can assume that $\int_{\Omega \cap B_{R_{g}}}|u(x, T)|^{2} d x \neq 0$. Let $\theta$ be given in Lemma 3.2 and $R_{0}:=\left(1+2 \delta_{g}\right) R_{g}$. Let $\sigma_{0} \in C_{0}^{\infty}\left(\mathbb{R}^{n}\right)$ satisfy that

$$
\operatorname{supp} \sigma_{0} \subset B_{R_{0}}, 0 \leq \sigma_{0} \leq 1 \text {, and } \sigma_{0}=1 \text { on } B_{\left(1+3 \delta_{g} / 2\right) R_{g}} \text {. }
$$

Write $\phi:=\sigma_{0} \cdot u$. We organize the rest of the proof in several steps.

Step 1. We present several observations on the truncated function $\phi$.

Observation One: By direct computations, we find

$$
\phi_{t}-\triangle \phi=-\nabla \phi \cdot \nabla v-\phi \triangle v+u \nabla v \cdot \nabla \sigma_{0}-2 \nabla u \cdot \nabla \sigma_{0}-u \triangle \sigma_{0}, \quad x \in \Omega, t \in[0, T] .
$$

Observation Two: If we set

$$
\psi:=u \nabla v \cdot \nabla \sigma_{0}-2 \nabla u \cdot \nabla \sigma_{0}-u \triangle \sigma_{0}, \quad x \in \Omega, t \in[0, T]
$$

then we have

$$
\psi(x, t)=0, \text { when } x \in B_{\left(1+3 \delta_{g} / 2\right) R_{g}}, t \in[0, T]
$$




$$
\int_{\Omega \cap B_{R_{0}}}|\psi(x, t)|^{2} d x \leq C_{1} M^{2}\left(1+t^{-1}\right) e^{L_{1} M^{2} t} \int_{\Omega}|u(x, 0)|^{2} d x, t \in(0, T],
$$

where $L_{1}$ is given in Theorem 2.2 and where $C_{1}>0$ is a constant (depending only on $\Omega, R_{g}$, and $\delta_{g}$ ). Indeed, (3.22) follows directly from (3.21) and (3.19). To show (3.23), we first notice that by (3.19), there is $C:=C\left(R_{g}, \delta_{g}\right)$ so that

$$
\left|\triangle \sigma_{0}(x)\right| \leq C\left(R_{g}, \delta_{g}\right) \text { and }\left|\nabla \sigma_{0}(x)\right| \leq C\left(R_{g}, \delta_{g}\right), \quad x \in \mathbb{R}^{n}
$$

we then use (3.21) and Cauchy's inequality to get that when $t \in[0, T]$,

$$
\begin{aligned}
& \int_{\Omega \cap B_{R_{0}}}|\psi(x, t)|^{2} d x=\int_{\Omega \cap B_{R_{0}}}\left|\left[u \nabla v \cdot \nabla \sigma_{0}-2 \nabla u \cdot \nabla \sigma_{0}-u \triangle \sigma_{0}\right](x, t)\right|^{2} d x \\
\leq & \int_{\Omega \cap B_{R_{0}}}\left[\left(|u|^{2}+|\nabla u|^{2}+|u|^{2}\right)(x, t)\right]\left[\left(\left|\nabla v \cdot \nabla \sigma_{0}\right|^{2}+4\left|\nabla \sigma_{0}\right|^{2}+\left|\triangle \sigma_{0}\right|^{2}\right)(x, t)\right] d x ;
\end{aligned}
$$

we finally use the above inequality, (2.24), (3.24) and Theorem 2.2 to get (3.23).

Observation Three: Taking $z=\phi$ in (3.4), we see from Lemma 3.1 that

$$
\begin{aligned}
& \frac{1}{2} \frac{d}{d t} \int_{\Omega \cap B_{R_{0}}}|\phi(x, t)|^{2} G_{\lambda}(x, t) d x+N_{\lambda}(t) \int_{\Omega \cap B_{R_{0}}}|\phi(x, t)|^{2} G_{\lambda}(x, t) d x \\
= & \int_{\Omega \cap B_{R_{0}}} \phi\left(\partial_{t} \phi(x, t)-\triangle \phi(x, t)\right) G_{\lambda}(x, t) d x, \lambda>0, t \in[T-\theta, T]
\end{aligned}
$$

and

$$
\frac{d}{d t} N_{\lambda}(t) \leq \frac{1}{T-t+\lambda} N_{\lambda}(t)+\frac{\int_{\Omega \cap B_{R_{0}}}\left(\partial_{t} \phi(x, t)-\triangle \phi(x, t)\right)^{2} G_{\lambda} d x}{\int_{\Omega \cap B_{R_{0}}}|\phi(x, t)|^{2} G_{\lambda}(x, t) d x}, \lambda>0, t \in[T-\theta, T] .
$$

To show these, we arbitrarily fix $\lambda>0$. We claim

$$
\int_{\Omega \cap B_{R_{0}}}|\phi(x, t)|^{2} G_{\lambda}(x, t) d x \neq 0, \quad t \in[T-\theta, T] .
$$

Indeed, by (3.19), the fact that $\phi:=\sigma_{0} \cdot u$, and $R_{0}:=\left(1+2 \delta_{g}\right) R_{g}$, we can easily see

$$
\int_{\Omega \cap B_{R_{0}}}|\phi(x, t)|^{2} G_{\lambda}(x, t) d x \geq \int_{\Omega \cap B_{(1+\delta g) R_{g}}}|u(x, t)|^{2} G_{\lambda}(x, t) d x, \quad t \in[0, T] .
$$

Since $\int_{\Omega \cap B_{R_{g}}}|u(x, T)|^{2} d x \neq 0$, it follows from Remark 3.2 and (3.3) that when $t \in[T-\theta, T]$,

$$
\int_{\Omega \cap B_{\left(1+\delta_{g}\right) R_{g}}}|u(x, t)|^{2} G_{\lambda}(x, t) d x \geq \frac{1}{(T-t+\lambda)^{n / 2}} e^{-\frac{\left(1+\delta_{g}\right)^{2} R_{g}^{2}}{4(T-t+\lambda)}} \int_{\Omega \cap B_{\left(1+\delta_{g}\right) R_{g}}}|u(x, t)|^{2} d x>0,
$$

which, together with (3.28), leads to (3.27). Next, by (3.27), we can use Lemma 3.1, where $z$ is replaced by $\phi$, to get (3.25) and (3.26).

Step 2. We show that for each $\lambda>0$ and $\varepsilon \in(0, \theta)$,

$$
\frac{\int_{\Omega \cap B_{R_{0}}}|\psi(x, t)|^{2} G_{\lambda}(x, t) d x}{\int_{\Omega \cap B_{R_{0}}}|\phi(x, t)|^{2} G_{\lambda}(x, t) d x} \leq 2 C_{1} M^{2}\left(1+\frac{1}{T}\right) e^{L_{1} M^{2} T} e^{\frac{L_{2}}{\theta}} e^{-\frac{L_{6}}{\varepsilon+\lambda}}, \quad t \in[T-\varepsilon, T],
$$


where

$$
L_{6}:=-\frac{\left(1+\delta_{g}\right)^{2} R_{g}^{2}}{4}+\frac{\left(1+3 \delta_{g} / 2\right)^{2} R_{g}^{2}}{4},
$$

and where $L_{2}$ is given in Theorem 2.2.

To this end, we arbitrarily fix $\lambda>0$ and $\varepsilon \in(0, \theta)$. By (3.6), we have $0<\varepsilon<\theta<\min \left\{1, \frac{T}{2}\right\}$, from which, it follows that

$$
t^{-1} \leq \frac{2}{T}, \text { when } t \in[T-\varepsilon, T] .
$$

Meanwhile, by (3.22) and (3.28), we see that when $t \in[T-\varepsilon, T]$,

$$
\frac{\int_{\Omega \cap B_{R_{0}}}|\psi(x, t)|^{2} G_{\lambda}(x, t) d x}{\int_{\Omega \cap B_{R_{0}}}|\phi(x, t)|^{2} G_{\lambda}(x, t) d x} \leq \frac{\int_{\Omega \cap\left(B_{R_{0}} \backslash B_{\left(1+3 \delta_{g} / 2\right) R_{g}}\right)}|\psi(x, t)|^{2} G_{\lambda}(x, t) d x}{\int_{\Omega \cap B_{\left(1+\delta_{g}\right) R_{g}}}|u(x, t)|^{2} G_{\lambda}(x, t) d x},
$$

which, along with (3.3) and (3.30), yields that when $t \in[T-\varepsilon, T]$,

$$
\frac{\int_{\Omega \cap B_{R_{0}}}|\psi(x, t)|^{2} G_{\lambda}(x, t) d x}{\int_{\Omega \cap B_{R_{0}}}|\phi(x, t)|^{2} G_{\lambda}(x, t) d x} \leq \frac{\int_{\Omega \cap\left(B_{R_{0}} \backslash B_{\left(1+3 \delta_{g} / 2\right) R_{g}}\right)}|\psi(x, t)|^{2} d x}{\int_{\Omega \cap B_{\left(1+\delta_{g}\right) R_{g}}}|u(x, t)|^{2} d x} e^{-\frac{L_{6}}{T-t+\lambda}} .
$$

This, together with (3.23), shows that when $t \in[T-\varepsilon, T]$,

$$
\frac{\int_{\Omega \cap B_{R_{0}}}|\psi(x, t)|^{2} G_{\lambda}(x, t) d x}{\int_{\Omega \cap B_{R_{0}}}|\phi(x, t)|^{2} G_{\lambda}(x, t) d x} \leq \frac{C_{1} M^{2}\left(1+t^{-1}\right) e^{L_{1} M^{2} t} \int_{\Omega}|u(x, 0)|^{2} d x}{\int_{\Omega \cap B_{\left(1+\delta_{g}\right) R_{g}}}|u(x, t)|^{2} d x} e^{-\frac{L_{6}}{\varepsilon+\lambda}} .
$$

Now, by (3.32), Lemma 3.2, and (3.31), we see that (3.29) holds for all $t \in[T-\varepsilon, T]$.

Step 3. We show that for any $\lambda>0$ and $\varepsilon \in(0, \theta)$,

$$
\begin{aligned}
& \lambda N_{\lambda}(T) \leq \frac{2(\lambda+\varepsilon)}{\varepsilon} \cdot \exp \left(C_{2} M^{2} \varepsilon\right) \\
& \times \quad\left[\frac{C_{3}}{2} M^{2} \varepsilon+\frac{C_{2}}{2} M^{2} \varepsilon^{2}+\frac{\varepsilon}{2} Q(\theta, \varepsilon, \lambda)+\frac{\left(1+\delta_{g}\right)^{2} R_{g}^{2}}{2 \varepsilon}+L_{1} M^{2} T+\frac{L_{2}}{\theta}\right],
\end{aligned}
$$

where $C_{2}, C_{3}$ are positive constants depending only on $\Omega$, and where

$$
Q(\theta, \varepsilon, \lambda):=6 C_{1} M^{2}\left(1+\frac{1}{T}\right) e^{L_{1} M^{2} T} e^{\frac{L_{2}}{\theta}} e^{-\frac{L_{6}}{\varepsilon+\lambda}}(1+\varepsilon) .
$$

Arbitrarily fix $\lambda>0$ and $\varepsilon \in(0, \theta)$. It follows from (3.20), (3.26), and the second equation of (1.1) that when $t \in[T-\varepsilon, T]$,

$$
\frac{d}{d t} N_{\lambda}(t) \leq \frac{1}{T-t+\lambda} N_{\lambda}(t)+\frac{\int_{\Omega \cap B_{R_{0}}}[(-\nabla \phi \cdot \nabla v-\phi(a v-b u)+\psi)(x, t)]^{2} G_{\lambda}(x, t) d x}{\int_{\Omega \cap B_{R_{0}}}|\phi(x, t)|^{2} G_{\lambda}(x, t) d x} .
$$

This, along with Cauchy's inequality, (2.25), and (2.24), yields that when $t \in[T-\varepsilon, T]$,

$$
\frac{d}{d t} N_{\lambda}(t) \leq \frac{1}{T-t+\lambda} N_{\lambda}(t)+\frac{\int_{\Omega \cap B_{R_{0}}} 3\left(|\nabla \phi|^{2}|\nabla v|^{2}+|\phi|^{2}|(a v-b u)|^{2}+|\psi|^{2}\right)(x, t) G_{\lambda}(x, t) d x}{\int_{\Omega \cap B_{R_{0}}}|\phi(x, t)|^{2} G_{\lambda}(x, t) d x}
$$




$$
\leq\left(\frac{1}{T-t+\lambda}+C_{2} M^{2}\right) N_{\lambda}(t)+C_{2} M^{2}+3 \frac{\int_{\Omega \cap B_{R_{0}}}|\psi(x, t)|^{2} G_{\lambda}(x, t) d x}{\int_{\Omega \cap B_{R_{0}}}|\phi(x, t)|^{2} G_{\lambda}(x, t) d x},
$$

for some $C_{2}>0$ depending only on $\Omega$. It, together with (3.29), implies that when $t \in[T-\varepsilon, T]$,

$$
\frac{d}{d t} N_{\lambda}(t)-\left(\frac{1}{T-t+\lambda}+C_{2} M^{2}\right) N_{\lambda}(t) \leq C_{2} M^{2}+6 C_{1} M^{2}\left(1+\frac{1}{T}\right) e^{L_{1} M^{2} T} e^{\frac{L_{2}}{\theta}} e^{-\frac{L_{6}}{\varepsilon+\lambda}} .
$$

Multiplying the above by $\exp \left(\ln (T-t+\lambda)-C_{2} M^{2} t\right)$, and then integrating it over $(t, T)$, we obtain

$$
\begin{aligned}
& \lambda \exp \left(-C_{2} M^{2} T\right) N_{\lambda}(T) \leq(T-t+\lambda) \exp \left(-C_{2} M^{2} t\right) N_{\lambda}(t) \\
+ & \left(C_{2} M^{2}+6 C_{1} M^{2}\left(1+\frac{1}{T}\right) e^{L_{1} M^{2} T} e^{\frac{L_{2}}{\theta}} e^{-\frac{L_{6}}{\varepsilon+\lambda}}\right) \int_{t}^{T} e^{-C_{2} M^{2} s}(T-s+\lambda) d s, \quad t \in[T-\varepsilon, T] .
\end{aligned}
$$

Dividing both sides of the above by $\exp \left(-C_{2} M^{2} T\right)$, we see that when $t \in[T-\varepsilon, T]$,

$$
\begin{aligned}
& \lambda N_{\lambda}(T) \leq(T-t+\lambda) \exp \left(C_{2} M^{2}(T-t)\right) N_{\lambda}(t) \\
+ & \left(C_{2} M^{2}+6 C_{1} M^{2}\left(1+\frac{1}{T}\right) e^{L_{1} M^{2} T} e^{\frac{L_{2}}{\theta}} e^{-\frac{L_{6}}{\varepsilon+\lambda}}\right) \int_{t}^{T} e^{C_{2} M^{2}(T-s)}(T-s+\lambda) d s \\
\leq & (\lambda+\varepsilon) \exp \left(C_{2} M^{2} \varepsilon\right)\left[N_{\lambda}(t)+\left(C_{2} M^{2}+6 C_{1} M^{2}\left(1+\frac{1}{T}\right) e^{L_{1} M^{2} T} e^{\frac{L_{2}}{\theta}} e^{-\frac{L_{6}}{\varepsilon+\lambda}}\right) \varepsilon\right] .
\end{aligned}
$$

This implies that when $t \in[T-\varepsilon, T]$,

$$
\frac{\lambda}{\lambda+\varepsilon} \exp \left(-C_{2} M^{2} \varepsilon\right) N_{\lambda}(T)-\left(C_{2} M^{2}+6 C_{1} M^{2}\left(1+\frac{1}{T}\right) e^{L_{1} M^{2} T} e^{\frac{L_{2}}{\theta}} e^{-\frac{L_{6}}{\varepsilon+\lambda}}\right) \varepsilon \leq N_{\lambda}(t) .
$$

Meanwhile, it follows from (3.20) and (3.25) that

$$
\begin{aligned}
& \frac{1}{2} \frac{d}{d t} \int_{\Omega \cap B_{R_{0}}}|\phi(x, t)|^{2} G_{\lambda}(x, t) d x+N_{\lambda}(t) \int_{\Omega \cap B_{R_{0}}}|\phi(x, t)|^{2} G_{\lambda}(x, t) d x \\
= & \int_{\Omega \cap B_{R_{0}}} \phi(x, t)[-\nabla \phi \cdot \nabla v-\phi \triangle v+\psi](x, t) G_{\lambda}(x, t) d x, \quad t \in[T-\varepsilon, T] .
\end{aligned}
$$

This, along with the second equation of (1.1), (2.24), (2.25), and Cauchy's inequality, indicates that when $t \in[T-\varepsilon, T]$,

$$
\begin{aligned}
& \frac{1}{2} \frac{d}{d t} \int_{\Omega \cap B_{R_{0}}}|\phi(x, t)|^{2} G_{\lambda}(x, t) d x+N_{\lambda}(t) \int_{\Omega \cap B_{R_{0}}}|\phi(x, t)|^{2} G_{\lambda}(x, t) d x \\
\leq & \frac{1}{2} \int_{\Omega \cap B_{R_{0}}}|\nabla \phi(x, t)|^{2} G_{\lambda}(x, t) d x+\frac{1}{2} \int_{\Omega \cap B_{R_{0}}}|\phi(x, t) \nabla v(x, t)|^{2} G_{\lambda}(x, t) d x \\
+ & \int_{\Omega \cap B_{R_{0}}}\left[\phi^{2}(b u-a v)+\phi \psi\right](x, t) G_{\lambda}(x, t) d x \\
\leq & \left(\frac{1}{2} N_{\lambda}(t)+C_{3} M^{2}\right) \int_{\Omega \cap B_{R_{0}}}|\phi(x, t)|^{2} G_{\lambda}(x, t) d x+\int_{\Omega \cap B_{R_{0}}}|\psi(x, t)|^{2} G_{\lambda}(x, t) d x,
\end{aligned}
$$

where $C_{3}$ is a positive constant depending only on $\Omega$. Then, after some computations, we obtain that when $t \in[T-\varepsilon, T]$,

$$
\begin{aligned}
& \frac{1}{2} \frac{d}{d t} \int_{\Omega \cap B_{R_{0}}}|\phi(x, t)|^{2} G_{\lambda}(x, t) d x+\frac{1}{2} N_{\lambda}(t) \int_{\Omega \cap B_{R_{0}}}|\phi(x, t)|^{2} G_{\lambda}(x, t) d x \\
\leq & \left(C_{3} M^{2}+\frac{\int_{\Omega \cap B_{R_{0}}}|\psi(x, t)|^{2} G_{\lambda}(x, t) d x}{\int_{\Omega \cap B_{R_{0}}}|\phi(x, t)|^{2} G_{\lambda}(x, t) d x}\right) \int_{\Omega \cap B_{R_{0}}}|\phi(x, t)|^{2} G_{\lambda}(x, t) d x .
\end{aligned}
$$


Thus, by (3.36), (3.35), and (3.29), we find that when $t \in[T-\varepsilon, T]$,

$$
\begin{aligned}
& \frac{d}{d t} \int_{\Omega \cap B_{R_{0}}}|\phi(x, t)|^{2} G_{\lambda}(x, t) d x+\frac{\lambda}{\lambda+\varepsilon} \exp \left(-C_{2} M^{2} \varepsilon\right) N_{\lambda}(T) \int_{\Omega \cap B_{R_{0}}}|\phi(x, t)|^{2} G_{\lambda}(x, t) d x \\
\leq & \left(C_{3} M^{2}+C_{2} M^{2} \varepsilon+6 C_{1} M^{2}\left(1+\frac{1}{T}\right) e^{L_{1} M^{2} T} e^{\frac{L_{2}}{\theta}} e^{-\frac{L_{6}}{\varepsilon+\lambda}}(1+\varepsilon)\right) \int_{\Omega \cap B_{R_{0}}}|\phi(x, t)|^{2} G_{\lambda}(x, t) d x .
\end{aligned}
$$

This, along with (3.34), yields that when $t \in[T-\varepsilon, T]$,

$$
\frac{d}{d t}\left(e^{\left(\frac{\lambda}{\lambda+\varepsilon} \exp \left(-C_{2} M^{2} \varepsilon\right) N_{\lambda}(T)-C_{3} M^{2}-C_{2} M^{2} \varepsilon-Q(\theta, \varepsilon, \lambda)\right) t} \int_{\Omega \cap B_{R_{0}}}|\phi(x, t)|^{2} G_{\lambda}(x, t) d x\right) \leq 0 .
$$

Integrating it over $\left(T-\varepsilon, T-\frac{\varepsilon}{2}\right)$, we have

$$
\begin{aligned}
& e^{\frac{\lambda}{\lambda+\varepsilon} \exp \left(-C_{2} M^{2} \varepsilon\right) N_{\lambda}(T) \frac{\varepsilon}{2}} \int_{\Omega \cap B_{R_{0}}}\left|\phi\left(x, T-\frac{\varepsilon}{2}\right)\right|^{2} G_{\lambda}\left(x, T-\frac{\varepsilon}{2}\right) d x \\
\leq & e^{\left(C_{3} M^{2}+C_{2} M^{2} \varepsilon+Q(\theta, \varepsilon, \lambda)\right) \frac{\varepsilon}{2}} \int_{\Omega \cap B_{R_{0}}}|\phi(x, T-\varepsilon)|^{2} G_{\lambda}(x, T-\varepsilon) d x,
\end{aligned}
$$

from which, it follows that

$$
\begin{aligned}
& e^{\frac{\varepsilon}{2(\lambda+\varepsilon)} \exp \left(-C_{2} M^{2} \varepsilon\right) \lambda N_{\lambda}(T)} \\
\leq & e^{\left(C_{3} M^{2}+C_{2} M^{2} \varepsilon+Q(\theta, \varepsilon, \lambda)\right) \frac{\varepsilon}{2}} \frac{\int_{\Omega \cap B_{R_{0}}}|\phi(x, T-\varepsilon)|^{2} G_{\lambda}(x, T-\varepsilon) d x}{\int_{\Omega \cap B_{R_{0}}}\left|\phi\left(x, T-\frac{\varepsilon}{2}\right)\right|^{2} G_{\lambda}\left(x, T-\frac{\varepsilon}{2}\right) d x} .
\end{aligned}
$$

We next estimate the right hand side of (3.37). One can directly check

$$
\begin{aligned}
\frac{\int_{\Omega \cap B_{R_{0}}}|\phi(x, T-\varepsilon)|^{2} G_{\lambda}(x, T-\varepsilon) d x}{\int_{\Omega \cap B_{R_{0}}}\left|\phi\left(x, T-\frac{\varepsilon}{2}\right)\right|^{2} G_{\lambda}\left(x, T-\frac{\varepsilon}{2}\right) d x} & \leq \frac{\int_{\Omega \cap B_{R_{0}}}|\phi(x, T-\varepsilon)|^{2} e^{-\frac{\left|x-x_{g}\right|^{2}}{4(\varepsilon+\lambda)}} d x}{\int_{\Omega \cap B_{R_{0}}}\left|\phi\left(x, T-\frac{\varepsilon}{2}\right)\right|^{2} e^{-\frac{\left|x-x_{g}\right|^{2}}{4(\varepsilon / 2+\lambda)}} d x} \\
& \leq \frac{\int_{\Omega \cap B_{R_{0}}}|\phi(x, T-\varepsilon)|^{2} d x}{e^{-\frac{\left(1+\delta_{g}\right)^{2} R_{g}^{2}}{2 \varepsilon}} \int_{\Omega \cap B_{\left(1+\delta_{g}\right) R_{g}}}\left|\phi\left(x, T-\frac{\varepsilon}{2}\right)\right|^{2} d x} .
\end{aligned}
$$

At the same time, by (3.19) and $\phi:=\sigma_{0} \cdot u$, we get

$$
\frac{\int_{\Omega \cap B_{R_{0}}}|\phi(x, T-\varepsilon)|^{2} d x}{\int_{\Omega \cap B_{\left(1+\delta_{g}\right) R_{g}}}\left|\phi\left(x, T-\frac{\varepsilon}{2}\right)\right|^{2} d x} \leq \frac{\int_{\Omega \cap B_{R_{0}}}|u(x, T-\varepsilon)|^{2} d x}{\int_{\Omega \cap B_{\left(1+\delta_{g}\right) R_{g}}\left|u\left(x, T-\frac{\varepsilon}{2}\right)\right|^{2} d x} .}
$$

Then, by Theorem 2.2. Lemma 3.2 and the fact that $\varepsilon \in(0, \theta)$, we see that

$$
\frac{\int_{\Omega \cap B_{R_{0}}}|\phi(x, T-\varepsilon)|^{2} d x}{\int_{\Omega \cap B_{\left(1+\delta_{g}\right) R_{g}}}\left|\phi\left(x, T-\frac{\varepsilon}{2}\right)\right|^{2} d x} \leq \frac{e^{L_{1} M^{2} T} \int_{\Omega}|u(x, 0)|^{2} d x}{\int_{\Omega \cap B_{\left(1+\delta_{g}\right) R_{g}}}\left|u\left(x, T-\frac{\varepsilon}{2}\right)\right|^{2} d x} \leq e^{L_{1} M^{2} T} e^{\frac{L_{2}}{\theta}} .
$$

This, together with (3.37) and (3.38), yields

$$
e^{\frac{\varepsilon}{2(\lambda+\varepsilon)} \exp \left(-C_{2} M^{2} \varepsilon\right) \lambda N_{\lambda}(T)} \leq e^{\left(C_{3} M^{2}+C_{2} M^{2} \varepsilon+Q(\theta, \varepsilon, \lambda)\right) \frac{\varepsilon}{2}} e^{\frac{\left(1+\delta_{g}\right)^{2} R_{g}^{2}}{2 \varepsilon}} e^{L_{1} M^{2} T} e^{\frac{L_{2}}{\theta}},
$$

which gives (3.33). 
Step 4. We prove (3.2) via tuning parameters.

Let $\varepsilon=k \theta$ and $\lambda=\mu \varepsilon$, where $k:=\min \left\{\frac{L_{6}}{2 L_{2}}, \frac{1}{2}\right\}$ and $\mu \in(0,1)$ will be determined later. Then we have $L_{2}-\frac{L_{6}}{k(1+\mu)}<0$. This, together with (3.34) (where $\varepsilon=k \theta$ and $\lambda=\mu \varepsilon$ ) and the fact that $0<\varepsilon<\theta<\min \left\{1, \frac{T}{2}\right\}$, indicates

$$
Q(\theta, \varepsilon, \lambda) \leq 12 C_{1} M^{2}\left(1+\frac{1}{T}\right) e^{L_{1} M^{2} T}
$$

Then, by (3.33) and the fact that $\lambda=\mu \varepsilon$, we see

$$
\begin{gathered}
\varepsilon \lambda N_{\lambda}(T) \leq 2(\mu+1) e^{C_{2} M^{2} \varepsilon}\left[\frac{C_{3}}{2} M^{2} \varepsilon^{2}+\frac{C_{2}}{2} M^{2} \varepsilon^{3}\right. \\
\left.+\quad 6 C_{1} M^{2}\left(1+\frac{1}{T}\right) e^{L_{1} M^{2} T} \varepsilon^{2}+\frac{\left(1+\delta_{g}\right)^{2} R_{g}^{2}}{2}+L_{1} M^{2} T \varepsilon+\frac{L_{2} \varepsilon}{\theta}\right] .
\end{gathered}
$$

Since $\frac{\varepsilon}{\theta}=k$ and $\varepsilon, k, \mu \in(0,1)$, we have

$$
\varepsilon \lambda N_{\lambda}(T) \leq 4 e^{C_{2} M^{2}}\left[\frac{C_{3}}{2} M^{2}+\frac{C_{2}}{2} M^{2}+6 C_{1} M^{2}\left(1+\frac{1}{T}\right) e^{L_{1} M^{2} T}+\frac{\left(1+\delta_{g}\right)^{2} R_{g}^{2}}{2}+L_{1} M^{2} T+L_{2}\right] .
$$

From the above, we can find a constant $C_{0}>1$, depending on $\Omega, r, R_{g}, \delta_{g}, M$, and $T$, so that

$$
\frac{16 \lambda}{r^{2}}\left(\lambda N_{\lambda}(T)+\frac{n}{4}\right)=\frac{16 \mu \varepsilon}{r^{2}}\left(\lambda N_{\lambda}(T)+\frac{n}{4}\right) \leq \frac{16}{r^{2}} \mu\left(\varepsilon \lambda N_{\lambda}(T)+\frac{n}{4}\right) \leq \mu C_{0} .
$$

Choosing $\mu=\frac{1}{2 C_{0}} \in(0,1)$ in (3.39), we obtain

$$
\frac{16 \lambda}{r^{2}}\left(\lambda N_{\lambda}(T)+\frac{n}{4}\right) \leq \frac{1}{2} .
$$

Next, since $0<r<R_{g}$ and $B_{r}:=B\left(x_{g}, r\right) \subset \Omega$, we find

$$
\begin{aligned}
& \int_{\Omega \cap B_{R_{0}}}|\phi(x, T)|^{2} e^{-\frac{\left|x-x_{g}\right|^{2}}{4 \lambda}} d x \\
= & \int_{\Omega \cap B_{R_{0}} \backslash B_{r}}|\phi(x, T)|^{2} e^{-\frac{\left|x-x_{g}\right|^{2}}{4 \lambda}} d x+\int_{B_{r}}|\phi(x, T)|^{2} e^{-\frac{\left|x-x_{g}\right|^{2}}{4 \lambda}} d x \\
\leq & \frac{1}{r^{2}} \int_{\Omega \cap B_{R_{0}} \backslash B_{r}}\left|x-x_{g}\right|^{2}|\phi(x, T)|^{2} e^{-\frac{\left|x-x_{g}\right|^{2}}{4 \lambda}} d x+\int_{B_{r}}|\phi(x, T)|^{2} e^{-\frac{\left|x-x_{g}\right|^{2}}{4 \lambda}} d x .
\end{aligned}
$$

Meanwhile, it follows from Lemma 3.3 that

$$
\begin{aligned}
& \int_{\Omega \cap B_{R_{0}}}\left|x-x_{g}\right|^{2}|\phi(x, T)|^{2} e^{-\frac{\left|x-x_{g}\right|^{2}}{4 \lambda}} d x \\
\leq & 8 \lambda\left(2 \lambda \int_{\Omega \cap B_{R_{0}}}|\nabla \phi(x, T)|^{2} e^{-\frac{\left|x-x_{g}\right|^{2}}{4 \lambda}} d x+\frac{n}{2} \int_{\Omega \cap B_{R_{0}}}|\phi(x, T)|^{2} e^{-\frac{\left|x-x_{g}\right|^{2}}{4 \lambda}} d x\right) \\
\leq & 8 \lambda\left(2 \lambda N_{\lambda}(T) \int_{\Omega \cap B_{R_{0}}}|\phi(x, T)|^{2} e^{-\frac{\left|x-x_{g}\right|^{2}}{4 \lambda}} d x+\frac{n}{2} \int_{\Omega \cap B_{R_{0}}}|\phi(x, T)|^{2} e^{-\frac{\left|x-x_{g}\right|^{2}}{4 \lambda}} d x\right) .
\end{aligned}
$$

Combining the above with (3.41) yields

$$
\int_{\Omega \cap B_{R_{0}}}|\phi(x, T)|^{2} e^{-\frac{\left|x-x_{g}\right|^{2}}{4 \lambda}} d x
$$




$$
\leq \frac{16 \lambda}{r^{2}}\left(\lambda N_{\lambda}(T)+\frac{n}{4}\right) \int_{\Omega \cap B_{R_{0}}}|\phi(x, T)|^{2} e^{-\frac{\left|x-x_{g}\right|^{2}}{4 \lambda}} d x+\int_{B_{r}}|\phi(x, T)|^{2} e^{-\frac{\left|x-x_{g}\right|^{2}}{4 \lambda}} d x .
$$

This, along with (3.40), implies

$$
\int_{\Omega \cap B_{R_{0}}}|\phi(x, T)|^{2} e^{-\frac{\left|x-x_{g}\right|^{2}}{4 \lambda}} d x \leq 2 \int_{B_{r}}|\phi(x, T)|^{2} e^{-\frac{\left|x-x_{g}\right|^{2}}{4 \lambda}} d x .
$$

Now, we are going to prove (3.2). One can easily check from (3.19) and $\phi:=\sigma_{0} \cdot u$ that

$$
\int_{\Omega \cap B_{R_{g}}}|u(x, T)|^{2} d x \leq e^{\frac{R_{g}^{2}}{4 \lambda}} \int_{\Omega \cap B_{R_{0}}}|\phi(x, T)|^{2} e^{-\frac{\left|x-x_{g}\right|^{2}}{4 \lambda}} d x .
$$

This, along with (3.42) and (3.19), shows

$$
\int_{\Omega \cap B_{R_{g}}}|u(x, T)|^{2} d x \leq 2 e^{\frac{R_{g}^{2}}{4 \lambda}} \int_{B_{r}}|\phi(x, T)|^{2} e^{-\frac{\left|x-x_{g}\right|^{2}}{4 \lambda}} d x \leq 2 e^{\frac{R_{g}^{2}}{4 \lambda}} \int_{B_{r}}|u(x, T)|^{2} d x .
$$

Since $\lambda=\mu \varepsilon=\mu k \theta$ (where $k=\min \left\{\frac{L_{6}}{2 L_{2}}, \frac{1}{2}\right\}$ and $\mu=\frac{1}{2 C_{0}}$ ), the above leads to

$$
\int_{\Omega \cap B_{R_{g}}}|u(x, T)|^{2} d x \leq 2 e^{\frac{1}{\theta} \frac{R_{g}^{2}}{4 \mu k}} \int_{B_{r}}|u(x, T)|^{2} d x .
$$

This, along with (3.6) and (3.7), indicates

$$
\int_{\Omega \cap B_{R_{g}}}|u(x, T)|^{2} d x \leq 2\left(e^{L_{4} M^{2} T} e^{L_{5}\left(1+\frac{1}{T}\right)} \frac{\mathbb{E}}{\int_{\Omega \cap B_{R_{g}}}|u(x, T)|^{2} d x}\right)^{\frac{L_{3} R_{g}^{2}}{4 \mu k}} \int_{B_{r}}|u(x, T)|^{2} d x .
$$

Meanwhile, according to Theorem 2.2 ,

$$
\mathbb{E} \leq\left(1+T e^{L_{1} M^{2} T}\right) \int_{\Omega}|u(x, 0)|^{2} d x \leq(1+T) e^{L_{1} M^{2} T} \int_{\Omega}|u(x, 0)|^{2} d x .
$$

This, together with (3.43), implies

$$
\int_{\Omega \cap B_{R_{g}}}|u(x, T)|^{2} d x \leq 2\left(D \frac{\int_{\Omega}|u(x, 0)|^{2} d x}{\int_{\Omega \cap B_{R_{g}}}|u(x, T)|^{2} d x}\right)^{\frac{L_{3} R_{g}^{2}}{4 \mu k}} \int_{B_{r}}|u(x, T)|^{2} d x,
$$

where $D=(1+T) e^{\left(L_{1}+L_{4}\right) M^{2} T+L_{5}\left(1+\frac{1}{T}\right)}$. The above leads to 13.2$)$ with $\gamma=\frac{L_{3} R_{g}^{2}}{4 \mu k+L_{3} R_{g}^{2}}$ (which depends only on $\Omega, r, R_{g}, \delta_{g}, M$, and $T$ ). This completes the proof of Theorem 3.1 .

Remark 3.3. It deserves mentioning that (3.2) is a local interpolation inequality of the boundary case for the system (1.1).

By the same argument used in Theorem 3.1, we can verify the local interpolation inequality of the interior case for the system (1.1): for each $p_{0} \in \Omega$, there is a positive number $r_{0}$, with $B\left(p_{0}, 3 r_{0}\right) \subseteq \Omega$. (Notice that the open ball $B\left(p_{0}, 3 r_{0}\right)$ is star-shaped with the center $\left.p_{0}.\right)$ Then, there are two constants $D=D\left(r_{0}, \Omega, T, M\right)>0$ and $\gamma=\gamma\left(r_{0}, \Omega, T, M\right) \in(0,1)$ so that

$$
\int_{B\left(p_{0}, r_{0}\right)}|u(x, T)|^{2} d x \leq\left(D \int_{\Omega}\left|u_{0}(x)\right|^{2} d x\right)^{\gamma}\left(2 \int_{B\left(p_{0}, r_{0} / 2\right)}|u(x, T)|^{2} d x\right)^{1-\gamma} .
$$

We omit the detailed proof. 


\section{Proof of the main results}

This section first proves Theorem 1.1, then it gives a qualitative unique continuation property for the system (1.1), as a consequence of Theorem 1.1 .

\subsection{Proof of Theorem 1.1}

Proof of Theorem 1.1. The proof will be organized in two steps.

Step 1. We prove (1.2), with the help of Thoerem 3.1.

Since $\omega$ is a nonempty open subset of $\Omega$, we can find $x_{0} \in \omega$ and $r>0$ so that the open ball $B\left(x_{0}, r\right)$ belongs to $\omega$. We are going to split the proof into two sub-steps.

Sub-step 1.1. We deal with the boundary of $\Omega$.

Since $\Omega$ is bounded domain with a $C^{2}$ boundary $\partial \Omega$, it follows from (3.1) that

$$
\partial \Omega \subset \cup_{g \in \partial \Omega} B\left(x_{g}, R_{g}\right) \text { and } \Omega \cap B\left(x_{g},\left(1+2 \delta_{g}\right) R_{g}\right) \text { is star-shaped with } x_{g},
$$

where the triplet $\left(x_{g}, R_{g}, \delta_{g}\right) \in \Omega \times \mathbb{R}^{+} \times(0,1]$ corresponding to $g \in \partial \Omega$ is given by (3.1). Then by the compactness of $\partial \Omega$, we can find a finite set of triplets $\left(x_{i}, R_{i}, \delta_{i}\right) \in \Omega \times \mathbb{R}^{+} \times(0,1]\left(i=1,2, \ldots, m_{1}\right)$ such that $\partial \Omega \subset \cup_{i=1,2, \ldots, m_{1}} B\left(x_{i}, R_{i}\right)$ and such that each $\Omega \cap B\left(x_{i},\left(1+2 \delta_{i}\right) R_{i}\right)$ is star-shaped with respect to $x_{i}$. Let

$$
\Theta_{1}=\cup_{i=1,2, \ldots, m_{1}} \Omega \cap B\left(x_{i}, R_{i}\right) .
$$

We claim that there exist two constants $D=D\left(\Theta_{1}, \Omega, r, M, T\right)>0$ and $\gamma_{1}=\gamma_{1}\left(\Theta_{1}, \Omega, r, M, T\right) \in$ $(0,1)$ such that

$$
\int_{\Theta_{1}}|u(x, T)|^{2} d x \leq D\left(\int_{\Omega}\left|u_{0}(x)\right|^{2} d x\right)^{\gamma_{1}}\left(\int_{B\left(x_{0}, r\right)}|u(x, T)|^{2} d x\right)^{1-\gamma_{1}} .
$$

In fact, for each $i \in\left\{1,2, \ldots, m_{1}\right\}$, we can choose $\rho_{i} \in\left(0, R_{i}\right)$ and finitely many points $q_{i, 1}, q_{i, 2}, \ldots, q_{i, d_{i}} \in \Omega$ so that

$$
\left\{\begin{array}{l}
x_{i}=q_{i, 1} ; \\
B\left(q_{i, j}, \rho_{i} / 2\right) \subset B\left(q_{i, j+1}, \rho_{i}\right), \forall j=1,2, \ldots, d_{i}-1 ; \\
B\left(q_{i, d_{i}}, \rho_{i}\right) \subset B\left(x_{0}, r\right) ; \\
B\left(q_{i, j}, 3 \rho_{i}\right) \subset \Omega, \forall j=1,2, \ldots, d_{i},
\end{array}\right.
$$

which forms a chain of balls along a curve connecting $q_{i, 1}$ with $q_{i, d_{i}}$. Then, it follows from Theorem 3.1 that there are constants $D_{i, 1}>0$ and $\alpha_{i, 1} \in(0,1)$ (which depend on $\rho_{i}, R_{i}, \delta_{i}, \Omega, M$, and $T$ ) so that

$$
\int_{\Omega \cap B\left(x_{i}, R_{i}\right)}|u(x, T)|^{2} d x \leq D_{i, 1}\left(\int_{\Omega}\left|u_{0}(x)\right|^{2} d x\right)^{\alpha_{i, 1}}\left(\int_{B\left(x_{i}, \rho_{i} / 2\right)}|u(x, T)|^{2} d x\right)^{1-\alpha_{i, 1}},
$$

which, along with the first fact in (4.3), yields

$$
\int_{\Omega \cap B\left(x_{i}, R_{i}\right)}|u(x, T)|^{2} d x \leq D_{i, 1}\left(\int_{\Omega}\left|u_{0}(x)\right|^{2} d x\right)^{\alpha_{i, 1}}\left(\int_{B\left(q_{i, 1}, \rho_{i} / 2\right)}|u(x, T)|^{2} d x\right)^{1-\alpha_{i, 1}} .
$$


Now, we will propagate the interpolation inequality (4.4) along the chain of balls (4.3). First, combining (4.4) with the second fact in (4.3) leads to

$$
\int_{\Omega \cap B\left(x_{i}, R_{i}\right)}|u(x, T)|^{2} d x \leq D_{i, 1}\left(\int_{\Omega}\left|u_{0}(x)\right|^{2} d x\right)^{\alpha_{i, 1}}\left(\int_{B\left(q_{i, 2}, \rho_{i}\right)}|u(x, T)|^{2} d x\right)^{1-\alpha_{i, 1}} .
$$

Next, we deal with the term $\int_{B\left(q_{i, 2}, \rho_{i}\right)}|u(x, T)|^{2} d x$ in (4.5) in the following manner: As mentioned in Remark 3.3. (Notice the fourth fact in (4.3).) there are two constants $C_{i, 2}=C_{i, 2}\left(\rho_{i}, \Omega, M, T\right)>0$ and $\beta_{i, 2}=\beta_{i, 2}\left(\rho_{i}, \Omega, M, T\right) \in(0,1)$ so that

$$
\int_{B\left(q_{i, 2}, \rho_{i}\right)}|u(x, T)|^{2} d x \leq C_{i, 2}\left(\int_{\Omega}\left|u_{0}(x)\right|^{2} d x\right)^{\beta_{i, 2}}\left(\int_{B\left(q_{i, 2}, \rho_{i} / 2\right)}|u(x, T)|^{2} d x\right)^{1-\beta_{i, 2}} .
$$

Combining (4.5) with (4.6) leads to

$$
\begin{aligned}
& \int_{\Omega \cap B\left(x_{i}, R_{i}\right)}|u(x, T)|^{2} d x \leq D_{i, 1}\left(\int_{\Omega}\left|u_{0}(x)\right|^{2} d x\right)^{\alpha_{i, 1}} \\
\times & \left(C_{i, 2}\left(\int_{\Omega}\left|u_{0}(x)\right|^{2} d x\right)^{\beta_{i, 2}}\left(\int_{B\left(q_{i, 2}, \rho_{i} / 2\right)}|u(x, T)|^{2} d x\right)^{1-\beta_{i, 2}}\right)^{1-\alpha_{i, 1}} \\
= & D_{i, 2}\left(\int_{\Omega}\left|u_{0}(x)\right|^{2} d x\right)^{\alpha_{i, 2}}\left(\int_{B\left(q_{i, 2}, \rho_{i} / 2\right)}|u(x, T)|^{2} d x\right)^{1-\alpha_{i, 2}},
\end{aligned}
$$

where $D_{i, 2}=D_{i, 1} \cdot C_{i, 2}^{1-\alpha_{i, 1}}>0$ and $\alpha_{i, 2}=\alpha_{i, 1}+\beta_{i, 2}\left(1-\alpha_{i, 1}\right) \in(0,1)$. Propagating interpolation inequalities finite times along the chain of balls (4.3), we can find constants $D_{i}=$ $D_{i}\left(\rho_{i}, R_{i}, \delta_{i}, \Omega, M, T\right)>0$ and $\alpha_{i}=\alpha_{i}\left(\rho_{i}, R_{i}, \delta_{i}, \Omega, M, T\right) \in(0,1)$ such that

$$
\int_{\Omega \cap B\left(x_{i}, R_{i}\right)}|u(x, T)|^{2} d x \leq D_{i}\left(\int_{\Omega}\left|u_{0}(x)\right|^{2} d x\right)^{\alpha_{i}}\left(\int_{B\left(q_{i, d_{i}-1}, \rho_{i} / 2\right)}|u(x, T)|^{2} d x\right)^{1-\alpha_{i}} .
$$

This, along with the second and the third fact of (4.3), yields that when $i \in\left\{1, \ldots, m_{1}\right\}$,

$$
\int_{\Omega \cap B\left(x_{i}, R_{i}\right)}|u(x, T)|^{2} d x \leq D_{i}\left(\int_{\Omega}\left|u_{0}(x)\right|^{2} d x\right)^{\alpha_{i}}\left(\int_{B\left(x_{0}, r\right)}|u(x, T)|^{2} d x\right)^{1-\alpha_{i}} .
$$

Let

$$
\gamma_{1}:=\max \left\{\alpha_{i} \mid i=1,2, \ldots, m_{1}\right\}
$$

We can easily check that $\gamma_{1} \in(0,1)$. This, together with (4.7), (4.8) and (2.26), implies that for each $i \in\left\{1, \ldots, m_{1}\right\}$,

$$
\begin{aligned}
& \int_{\Omega \cap B\left(x_{i}, R_{i}\right)}|u(x, T)|^{2} d x \leq D_{i}\left(\int_{\Omega}\left|u_{0}(x)\right|^{2} d x\right)^{\alpha_{i}} \\
\times & \left(\int_{B\left(x_{0}, r\right)}|u(x, T)|^{2} d x\right)^{1-\gamma_{1}}\left(e^{L_{1} M^{2} T} \int_{\Omega}\left|u_{0}(x)\right|^{2} d x\right)^{\gamma_{1}-\alpha_{i}} \\
= & \bar{D}_{i}\left(\int_{\Omega}\left|u_{0}(x)\right|^{2} d x\right)^{\gamma_{1}}\left(\int_{B\left(x_{0}, r\right)}|u(x, T)|^{2} d x\right)^{1-\gamma_{1}}
\end{aligned}
$$


where $\bar{D}_{i}=D_{i} \cdot e^{\left(\gamma_{1}-\alpha_{i}\right) L_{1} M^{2} T}>0$. Finally, by (4.1) and (4.9), we get (4.2), with $D:=\sum_{i=1}^{m_{1}} \bar{D}_{i}$ and $\gamma_{1}$ given by (4.8).

Sub-step 1.2. We deal with the interior of $\Omega$.

It is obvious that there exists a compact subset $\Theta_{2} \subset \Omega$ such that $\Omega \subseteq \Theta_{1} \cup \Theta_{2}$. By the compactness of $\Theta_{2}$, there is a constant $R>0$ and finitely many points $y_{1}, y_{2} \ldots, y_{m_{2}} \in \Omega$ such that $\Theta_{2} \subset \cup_{i=1,2, \ldots, m_{2}} B\left(y_{i}, R\right)$ and $B\left(y_{i}, 3 R\right) \subset \Omega$ for each $i \in\left\{1,2, \ldots, m_{2}\right\}$. Then, by the same method used to prove (4.2), we can find constants $D=D\left(\Theta_{2}, \Omega, r, M, T\right)>0$ and $\gamma_{2}=\gamma_{2}\left(\Theta_{2}, \Omega, r, M, T\right) \in$ $(0,1)$ so that

$$
\int_{\Theta_{2}}|u(x, T)|^{2} d x \leq D\left(\int_{\Omega}\left|u_{0}(x)\right|^{2} d x\right)^{\gamma_{2}}\left(\int_{B\left(x_{0}, r\right)}|u(x, T)|^{2} d x\right)^{1-\gamma_{2}} .
$$

Finally, by (4.2), (4.10), (2.25), and Lemma 2.1, we obtain (1.2).

Step 2. We prove (1.3).

The proof will also be split into two sub-steps.

Sub-step 2.1. We prove that $u(\cdot, t) \neq 0$ for each $t \in[0, T]$.

By contradiction, we suppose that $u(\cdot, t)=0$ for some $t \in(0, T]$. Then by the assumption that $u_{0} \neq 0$, we have that $0<T_{0} \leq T$, where

$$
T_{0}:=\inf \{t \in(0, T] \mid u(\cdot, t)=0\} .
$$

This, along with the continuity of $u$, yields

$$
u\left(\cdot, T_{0}\right)=0 \text { and } u(\cdot, t) \neq 0 \text {, for each } t \in\left[0, T_{0}\right) .
$$

Let

$$
\zeta(t):=\frac{\|u(\cdot, t)\|_{2}^{2}}{\|u(\cdot, t)\|_{H^{-1}}^{2}}, t \in\left[0, T_{0}\right)
$$

where $\|\cdot\|_{H^{-1}}$ is the norm of $H^{-1}(\Omega)$.

We now claim the following backward uniqueness estimate for $u$ :

$$
\left\|u_{0}\right\|_{H^{-1}}^{2} \leq \exp \left(2 e^{C(\Omega) M^{2} T}(\zeta(0)+C(\Omega) M \sqrt{\zeta(0)}) T\right)\|u(\cdot, t)\|_{H^{-1}}^{2} \text {, for each } t \in\left[0, T_{0}\right) .
$$

To this end, by multiplying the first equation of (1.1) by $u$ and $(-\triangle)^{-1} u$ respectively, and integrating them over $\Omega$, we obtain that for each $t \in[0, T]$,

$$
\left\{\begin{array}{l}
\frac{1}{2} \frac{d}{d t}\|u(\cdot, t)\|_{2}^{2}+\|u(\cdot, t)\|_{H_{0}^{1}}^{2}=\langle-\nabla(u(\cdot, t) \nabla v(\cdot, t)), u(\cdot, t)\rangle, \\
\frac{1}{2} \frac{d}{d t}\|u(\cdot, t)\|_{H^{-1}}^{2}+\|u(\cdot, t)\|_{2}^{2}=\left\langle-\nabla(u(\cdot, t) \nabla v(\cdot, t)),(-\triangle)^{-1} u(\cdot, t)\right\rangle_{H^{-1}, H_{0}^{1}} .
\end{array}\right.
$$

(Here, $\|\cdot\|_{H_{0}^{1}}$ denotes the norm of the space $H_{0}^{1}(\Omega),\langle\cdot, \cdot\rangle$ is the inner product in $L^{2}(\Omega)$, and $\langle\cdot, \cdot\rangle_{H^{-1}, H_{0}^{1}}$ stands for the pair between $H^{-1}(\Omega)$ and $H_{0}^{1}(\Omega)$.) Write

$$
f(x, t):=-\nabla(u(x, t) \nabla v(x, t)), \quad x \in \Omega, t \in[0, T] .
$$

By (4.15), (2.24), and (4.14), after direct computations, we get that

$$
\|f\|_{H^{-1}}=\|\nabla(u \nabla v)\|_{H^{-1}} \leq\|u \nabla v\|_{\left[L^{2}(\Omega)\right]^{n}}
$$




$$
\begin{gathered}
\leq\|u\|_{2}\|\nabla v\|_{\left[L^{\infty}(\Omega)\right]^{n}} \leq C(\Omega) M\|u\|_{2}, \quad t \in[0, T] \\
\zeta^{\prime}(t)=\frac{2}{\|u\|_{H^{-1}}^{4}}\left(\langle f, u\rangle\|u\|_{H^{-1}}^{2}-\|u\|_{H_{0}^{1}}^{2}\|u\|_{H^{-1}}^{2}\right. \\
\left.-\left\langle f,(-\Delta)^{-1} u\right\rangle_{H^{-1}, H_{0}^{1}}\|u\|_{2}^{2}+\|u\|_{2}^{4}\right), \quad t \in\left[0, T_{0}\right) ; \\
=\|u\|_{2}^{4}-\|u\|_{2}^{2}\left\langle f,(-\triangle)^{-1} u\right\rangle_{H^{-1}, H_{0}^{1}} \\
\leq\left|\left\langle\triangle u+f / 2,(-\triangle)^{-1} u\right\rangle_{H^{-1}, H_{0}^{1}}\right|^{2}-\left|\left\langle f / 2,(-\triangle)^{-1} u\right\rangle_{H^{-1}, H_{0}^{1}}\right|^{2} \\
=\left(\|u\|_{H_{0}^{1}}^{2}+\|f / 2\|_{H^{-1}}^{2}-\langle f, u\rangle\right)\|u\|_{H^{-1}}^{2}-\left|\left\langle f / 2,(-\triangle)^{-1} u\right\rangle_{H^{-1}, H_{0}^{1}}\right|^{2}, \quad t \in[0, T] .
\end{gathered}
$$

By (4.17) and (4.18), we see

$$
\zeta^{\prime}(t) \leq \frac{2}{\|u\|_{H^{-1}}^{2}}\|f / 2\|_{H^{-1}}^{2}, \quad t \in\left[0, T_{0}\right)
$$

which, together with (4.16), yields

$$
\zeta(t) \leq e^{C(\Omega) M^{2} t} \zeta(0), \quad t \in\left[0, T_{0}\right)
$$

Now, by the second equation in (4.14), (4.19), and (4.16), we have

$$
\begin{aligned}
0 & \leq \frac{1}{2} \frac{d}{d t}\|u\|_{H^{-1}}^{2}+\zeta(t)\|u\|_{H^{-1}}^{2}+\left|\left\langle f,(-\triangle)^{-1} u\right\rangle_{H^{-1}, H_{0}^{1}}\right| \\
& \leq \frac{1}{2} \frac{d}{d t}\|u\|_{H^{-1}}^{2}+\zeta(t)\|u\|_{H^{-1}}^{2}+\|f\|_{H^{-1}}\left\|(-\triangle)^{-1} u\right\|_{H_{0}^{1}} \\
& \leq \frac{1}{2} \frac{d}{d t}\|u\|_{H^{-1}}^{2}+\zeta(0) e^{C(\Omega) M^{2} t}\|u\|_{H^{-1}}^{2}+C(\Omega) M\|u\|_{2}\|u\|_{H^{-1}}, \quad t \in\left[0, T_{0}\right),
\end{aligned}
$$

which, along with (4.12) and (4.19), shows

$$
0 \leq \frac{1}{2} \frac{d}{d t}\|u\|_{H^{-1}}^{2}+e^{C(\Omega) M^{2} T}(\zeta(0)+C(\Omega) M \sqrt{\zeta(0)})\|u\|_{H^{-1}}^{2}, \quad t \in\left[0, T_{0}\right) .
$$

Multiplying the above by $\exp \left(2 e^{C(\Omega) M^{2} T}(\zeta(0)+C(\Omega) M \sqrt{\zeta(0)}) t\right)$, and then integrating it over $(0, t)$, where $t \in\left[0, T_{0}\right)$, we obtain (4.13).

Next, it follows from (4.13) that when $t \in\left[0, T_{0}\right)$,

$$
\begin{aligned}
& \frac{\left\|u_{0}\right\|_{2}^{2}}{\|u(\cdot, t)\|_{2}^{2}} \leq \frac{\left\|u_{0}\right\|_{H^{-1}}^{2}}{\|u(\cdot, t)\|_{H^{-1}}^{2}} \zeta(0) \\
\leq \quad & \zeta(0) \exp \left(2 e^{C(\Omega) M^{2} T}(\zeta(0)+C(\Omega) M \sqrt{\zeta(0)}) T\right) .
\end{aligned}
$$

Since $\zeta(0) \geq 1$, we have that $\sqrt{\zeta(0)} \leq \zeta(0)$ and $\zeta(0)<\exp (\zeta(0))$. These show that

$$
\frac{\left\|u_{0}\right\|_{2}^{2}}{\|u(\cdot, t)\|_{2}^{2}} \leq \exp \left(C(\Omega)(1+M T) e^{C(\Omega) M^{2} T} \zeta(0)\right), \quad t \in\left[0, T_{0}\right)
$$


Meanwhile, by $(i i)$ in Remark 2.3 and the assumption that $u_{0} \in L^{\infty}(\Omega)$, we see that $u \in$ $C\left([0, T] ; L^{2}(\Omega)\right)$. This, along with (4.11), implies that

$$
\lim _{t \rightarrow T_{0}^{-}}\|u(\cdot, t)\|_{2}^{2}=\left\|u\left(\cdot, T_{0}\right)\right\|_{2}^{2}=0
$$

which, together with the assumption that $u_{0} \neq 0$, contradicts (4.20). Hence, we finish the proof of Sub-step 2.1.

Sub-step 2.2. We prove (1.3).

According to Sub-step 2.1, the function $t \rightarrow \frac{\|u(\cdot, t)\|_{2}^{2}}{\|u(\cdot, t)\|_{H^{-1}}^{2}}$ (see (4.12) $)$ is well defined over [0,T]. We still use $\zeta(\cdot)$ to denote this function on $[0, T]$. Then by the same method in the proof of (4.20), we can verify that

$$
\left\|u_{0}\right\|_{2}^{2} \leq \exp \left(C(\Omega)(1+M T) e^{C(\Omega) M^{2} T} \zeta(0)\right)\|u(\cdot, T)\|_{2}^{2},
$$

which, together with (1.2), gives (1.3).

Hence, we finish the proof of Theorem 1.1 .

\subsection{Consequence of Theorem 1.1}

This subsection presents a qualitative unique continuation property for the system (1.1), which is a consequence of Theorem 1.1.

Corollary 4.1. Let $u_{0} \in L^{p}(\Omega)$ with $n<p \leq+\infty$ and let $\omega$ be a nonempty open subset of $\Omega$. Suppose that $(u, v)$ is the solution to the system (1.1) over $[0, T]$ for some $T>0$. Then

$$
u=0 \text { over } \Omega \times[0, T] \text { and } v=0 \text { over } \Omega \times(0, T],
$$

provided that either $u(\cdot, T)=0$ over $\omega$ or $v(\cdot, T)=0$ over $\omega$.

Proof. We organize the proof in two steps.

Step 1. We show the corollary when $p=+\infty$.

In the first case that $u(\cdot, T)=0$ over $\omega$, we can apply (1.3) to see that $u_{0}=0$, which, along with ( iii) of Remark 2.3, leads to (4.21) in this case.

We next consider the second case that $v(\cdot, T)=0$ over $\omega$. Multiplying the second equation of (1.1) by a test function $\chi \in C_{0}^{\infty}(\Omega)$ with supp $\chi \subset \omega$, we find

$$
\begin{aligned}
& \int_{\Omega} u(\cdot, T) \chi d x=\frac{1}{b} \int_{\Omega}[-\triangle v(\cdot, T)+a v(\cdot, T)] \chi d x \\
= & \frac{1}{b}\left[\int_{\Omega} a v(\cdot, T) \chi d x+\int_{\Omega} v(\cdot, T)(-\triangle \chi) d x\right]=0,
\end{aligned}
$$

which yields that $u(\cdot, T)=0$ over $\omega$, i.e., we return to the first case. Consequently, (4.21) is true for the second case.

Step 2. We show the corollary when $n<p<+\infty$. 
Arbitrarily fix $\epsilon \in(0, T)$. We define two functions $u_{\epsilon}$ and $v_{\epsilon}$ on $\Omega \times[0, T-\epsilon]$ by setting $u_{\epsilon}(x, t):=u(x, t+\epsilon)$ and $v_{\epsilon}(x, t):=v(x, t+\epsilon)$, respectively. It is obvious that $u_{\epsilon}$ and $v_{\epsilon}$ satisfy

$$
\begin{cases}u_{\epsilon t}-\triangle u_{\epsilon}(x, t)+\nabla \cdot\left(u_{\epsilon}(x, t) \nabla v_{\epsilon}(x, t)\right)=0, & \text { in } \Omega \times(0, T-\epsilon], \\ -\triangle v_{\epsilon}(x, t)+a v_{\epsilon}(x, t)-b u_{\epsilon}(x, t)=0, & \text { in } \Omega \times(0, T-\epsilon], \\ u_{\epsilon}(x, t)=0, v_{\epsilon}(x, t)=0, & \text { on } \partial \Omega \times(0, T-\epsilon], \\ u_{\epsilon}(x, 0)=u(x, \epsilon), & \text { in } \Omega .\end{cases}
$$

Since $u_{0} \in L^{p}(\Omega)(n<p<+\infty)$, it follows from the conclusion $(i i)$ in Theorem 2.1 that $u_{\epsilon}(\cdot, 0)=$ $u(\cdot, \epsilon) \in L^{\infty}(\Omega)$ and $\left(u_{\epsilon}, v_{\epsilon}\right) \in\left[L^{\infty}\left(0, T-\epsilon ; L^{\infty}(\Omega)\right)\right]^{2}$. Thus we can apply Theorem 1.1 (where $(u, v)$ is replaced by $\left.\left(u_{\epsilon}, v_{\epsilon}\right)\right)$ to see what follows:

(i) There are constants $\gamma=\gamma\left(\Omega, \omega,\left\|u_{\epsilon}(\cdot, 0)\right\|_{\infty}, T-\epsilon\right) \in(0,1)$ and $D=D\left(\Omega, \omega,\left\|u_{\epsilon}(\cdot, 0)\right\|_{\infty}, T-\epsilon\right)>$ 0 so that

$$
\int_{\Omega}\left|u_{\epsilon}(x, T-\epsilon)\right|^{2} d x \leq D\left(\int_{\Omega}\left|u_{\epsilon}(x, 0)\right|^{2} d x\right)^{1-\gamma}\left(\int_{\omega}\left|u_{\epsilon}(x, T-\epsilon)\right|^{2} d x\right)^{\gamma} .
$$

(ii) When $u(\cdot, \epsilon) \neq 0$, there is $C=C\left(\Omega, \omega,\left\|u_{\epsilon}(\cdot, 0)\right\|_{\infty}, T-\epsilon\right)>0$ so that

$$
\left.\int_{\Omega}\left|u_{\epsilon}(x, 0)\right|^{2} d x \leq C \exp \left(C \frac{\left\|u_{\epsilon}(x, 0)\right\|_{L^{2}(\Omega)}^{2}}{\left\|u_{\epsilon}(x, 0)\right\|_{H^{-1}(\Omega)}^{2}}\right) \times\left.\int_{\omega}\left(\mid u_{\epsilon}(x, T-\epsilon)\right)\right|^{2}\right) d x .
$$

Now, since $u(x, T)=0$ over $\omega$ or $v(x, T)=0$ over $\omega$, we have that $u_{\epsilon}(x, T-\epsilon)=0$ over $\omega$ or $v_{\epsilon}(x, T-\epsilon)=0$ over $\omega$. Thus, with the aid of the above $(i)$ and $(i i)$, we can use the result obtained in Step 1 (where $(u, v)$ is replaced by $\left.\left(u_{\epsilon}, v_{\epsilon}\right)\right)$ to get $u_{\epsilon}=v_{\epsilon}=0$ over $\Omega \times(0, T-\epsilon]$, i.e., $(u, v)=0$ over $\Omega \times(\epsilon, T]$. Since $\epsilon$ can be arbitrarily taken from $(0, T)$, we find that $(u, v)=0$ over $\Omega \times(0, T]$. This, along with the continuity of $u$, leads to (4.21). This ends the proof.

\section{References}

[1] S. Agmon, A. Douglis, L. Nirenberg, Estimates near the boundary for solutions of elliptic partial differential equations satisfying general boundary conditions I, Comm. Pure Appl. Math., 12(1959), 623-727.

[2] F.J. Almgren Jr., Dirichlet's problem for multiple valued functions and the regularity of mass minimizing integral currents, in "Minimal submanifolds and geodesics", 1-6, North-Holland, Amsterdam-New York, 1979.

[3] J. Apraiz, L. Escauriaza, G. Wang, C. Zhang, Observability inequalities and measurable sets. J. Eur. Math. Soc., 16(2014), 2433-2475.

[4] F.W. Chaves-Silva, S.Guerrero, A controllability result for a chemotaxis-fluid model, J. Differential Equations, 262(2017), 4863-4905.

[5] D. Daners, Heat kernal estimates for operators with boundary conditions, Math. Nachr., $217(2000), 13-41$. 
[6] J.L. Diaz, T. Nagai, Symmetrization in a parabolic-elliptic system related to chemotaxis. Adv. Math. Sci. Appl., 5 (1995), 659-680.

[7] L. Escauriaza, F.J. Fernández, Unique continuation for parabolic operators, Ark. Mat., 41(2003), 35-60.

[8] L. Escauriaza, F.J. Fernández, S. Vessella, Doubling properties of caloric functions, Appl. Anal., 85(2006), 205-223.

[9] B.Z. Guo, L. Zhang, Local null controllability for a chemotaxis system of parabolic-elliptic type, System \& Control Letters, 65(2014), 106-111.

[10] N. Garofalo, F. Lin, Monotonicity properties of variational integrals, $A_{p}$ weights and unique continuation. Indiana Univ. Math. J., 35(1986), 245-268.

[11] M.A. Herrero, J.J.L. Velázquez, Singularity patterns in a chemotaxis model. Math. Annalen, 306 (1996), 583-623.

[12] T. Hillen, K.J. Painter, A user's guide to PDE models for chemotaxis. J. Math. Biol., 58 (2009), 183-217.

[13] D. Horstmann, From 1970 until present: the Keller-Segel model in chemotaxis and its consequences I, Jahresber. Deutsch. Math. Verein., 105(2003), 103-165.

[14] E.F. Keller, L.A. Segel, Initiation of slime mode aggregation viewed as an instability, J. Theor. Biol., 26(1970), 399-415.

[15] Q. Huang, Y. Jin, M. Lewis, $R_{0}$ analysis of a benthic-drift model for a stream population, SIAM J. Appl. Dyna. Syst., 15(2016), 287-321.

[16] W. Jäger, S. Luckhaus, On explosions of solutions to a system of partial differential equations modelling chemotaxis, Trans. Amer. Math. Soc., 329(1992), 819-824.

[17] C.E. Kenig, Quantitative unique continuation, logarithmic convexity of Gaussian means and Hardy's uncertainty principle, Proc. Sympos. Pure Math., 79(2008), 207-227.

[18] C.E. Kenig, G. Ponce, L. Vega, On unique continuation for nonlinear Schrödinger equations, Comm. Pure Appl. Math., 56(2003), 1247-1262.

[19] H. Koch, D. Tataru, Carleman estimates and unique continuation for second order parabolic equations with nonsmooth coefficients, Comm. PDE, 34(2009), 305-366.

[20] I. Kukavica, Quantitative uniqueness for second-order elliptic operators, Duke Math. J., 91(1998), 225-240.

[21] E.M. Landis, O. A. Oleinik, Generalized analyticity and some related properties of solutions of elliptic and parabolic equations, Russian Mathematical Surveys, 29(1974), 195-212.

[22] F. Lin, A uniqueness theorem for parabolic equations. Comm. Pure Appl. Math., 43(1990), 127-136. 
[23] K.D. Phung, G. Wang, Quantitative unique continuation for the semilinear heat equation in a convex domain. J. Funct. Anal., 259(2010), 1230-1247.

[24] K.D. Phung, G. Wang, An observability estimate for parabolic equations from a measurable set in time and its applications. J. Eur. Math. Soc., 15(2013), 681-703.

[25] K.D. Phung, L. Wang, C. Zhang, Bang-bang property for time optimal control of semilinear heat equation, Ann. Inst. H. Poincaré Anal. Non Linéaire, 31(2014), 477-499.

[26] C. Poon, Unique continuation for parabolic equations, Comm. PDE, 21(1996), 521-539.

[27] S. Vessella, Unique continuation properties and quantitative estimates of unique continuation for parabolic equations, Handbook of Differential Equations: Evolutionary Equations 5, 2009, 421-500.

[28] H. Yamabe, A unique continuation theorem of a diffusion equation, Ann. of Math., 69(1959), 462-466.

[29] K. Yosida, Functional analysis 6th, Springger-Verlag, Berlin, 1980.

[30] B, Zhang, Unique continuation for the Korteweg-de Vries equation, Siam J. Math. Anal., 23(1992), 55-71. 